

\section{Public joursa or Transportation}

Gary L. Brosch, Editor

Patricia Henderson Ball, Managing Editor

\section{Editorial Board}

Robert B Cervero, Ph.D. University of California, Berkeley

Chester E. Colby MK Centennial

Gordon Fielding, Ph.D. University of Califormia, Irvine

David J. Forkenbrock, Ph.D.

University of lowa

José A. Gómez-Ibáñez, Ph.D.

Harvard University
Naomi W. Ledé, Ph.D.

Texas Southern University

William W. Millar American Public Transit Association

Steven E. Polzin, Ph.D., P.E. University of South Florida

Sandra Rosenbloom, Ph.D.

University of Arizona

Lawrence Schulman

Orbital Sciences Corp

George Smerk, D.B.A.

Indiana University

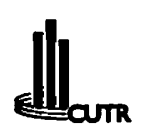

The Journal of Public Transportation (ISSN 1077-291X) is published quarterly by the Center for Urban Transportation Research (CUTR) in the College of Engineering at the University of South Florida. The contents of this document reflect the views of the authors, who are responsible for the facts and the accuracy of the information presented herein. This document is disseminated under the sponsorship of the U.S. Department of Transportation, University Research Institute Program, in the interest of information exchange. The U.S. Government assumes no liability for the contents or use thereof Subscriptions are complimentary and may be obtained by contacting the Center for Urban Transportation Research, University of South Florida, 4202 E. Fowler Avenue, CUT 100, Tampa, FL 33620-5375, (813) 974-3120, e-mail: pball@cutrengusfedu. 


\section{Public \\ JOURNAL OF \\ Transportation}

Volume 2, No. 4, 1999

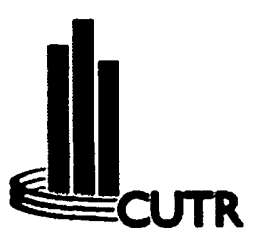

(C) 1999 Center for Urban Transportation Research

National Center for Transit Research

Center for Urban Transportation Research

College of Engineering - University of South Florida

4202 E. Fowler Avenue, CUT 100, Tampa, FL 33620-5375

(813) $974-3120$ - Fax (813) 974-5168

E-mail: pball@cutr.eng.usf.edu

Web Site: http://www.cutr.eng.usf.edu 



\section{Public \\ JOURNAL OF \\ Transportation}

Volume 2, No. 4, 1999

\section{Contents}

GIS-Based Support System for On-Demand Flexroute Transit Service ...... 1 Brian L. Smith, Priya K. Durvasula, and Stephen C. Brich

Planning and Analyzing Transit Networks: An Integrated Approach Regarding Requirements of Passengers and Operators

Markus Friedrich, Thomas Haupt, and Klaus Noekel

Transit GIS Applications in Fairfax County, Virginia 41 Wenyu Jia and Brendan Ford

A Transit Access Analysis of TANF Recipients in Portland, Oregon

Thomas W. Sanchez

Evaluating Transit Market Potential and Selecting Locations of Transit Service Facilities Using GIS

Srinivas S. Pulugurtha, Shashi S. Nambisan, and Nanda Srinivasan

Our troubled planet can no longer afford the luxury of pursuits confined to an ivory tower. Scholarship has to prove its worth, not on its own terms, but by service to the nation and the world.

- Oscar Handlin 



\section{Editorial}

We are pleased to present this special issue of the Journal of Public Transportation, which focuses on Geographic Information Systems (GIS) and includes five papers that were originally presented at the Second National GIS in Transit Conference. The conference, held on May 16-18, 1999, at the University of South Florida in Tampa, was a follow-up to the 1995 conference and focused on the use of GIS to support public transit planning, service delivery, and decision-making.

Produced in conjunction with the U.S. Department of Transportation's (USDOT) Volpe Center and sponsored by the National Center for Transit Research (NCTR) through the Research and Special Programs Administration (RSPA) at USDOT, the conference brought together transportation professionals from transit providers, academic institutions, the consulting industry, public agency transportation planners, and vendors of technology to exchange knowledge on the use of GIS in the support of the transit industry. More than 30 speakers participated in forums ranging from presentations to panels to workshops and vendor demonstrations to address the 170 attendees. Experts covered topics such as:

- evolving GIS capabilities;

- developing and applying GIS capabilities to public transportation;

- GIS applications to support paratransit service delivery;

- making GIS work;

- planning and implementing GIS systems;

- rural transit GIS applications;

- GIS applications in customer information;

- GIS applications in rail transit station area development;

- GIS applications in service planning;

- melding GIS into transit planning;

- GIS ingredients for public transit; and

- access to jobs.

Other organizations collaborating on program development for the conference included the Federal Highway Administration FHWA), the Association 
for Commuter Transportation (ACT), the American Public Transit Association (APTA), the Florida Department of Transportation (FDOT) Public Transit Office, the Bureau of Transportation Statistics (BTS), and the Association of American Geographers (AAG).

GIS is one of the tools by which the public transit industry can leverage technology and evolving information databases to improve the quality and efficiency in the delivery of transit services. GIS can be leveraged in an array of uses to support various aspects of how we plan, design, deliver, and administer public transit facilities and services. In an era where transit is striving to meet the growing mobility needs of the public while working with constrained resources and challenging demographic and geographic conditions, GIS is one of the tools that can help transit reach it potential.

We hope you find these papers useful and encourage you to peruse the proceedings of the Second National GIS in Transit Conference, which will be available on NCTR's web site (www.cutr.eng.usf.edu/nctr) in early 2000.

Steven E. Polzin, Ph.D., P.E. Conference Chairman 


\title{
GIS-Based Support System for On-Demand Flexroute Transit Service
}

\author{
Brian L. Smith, University of Virginia \\ Priya K. Durvasula, ALK Associates, Inc. \\ Stephen C. Brich, Virginia Transportation Research Council
}

\begin{abstract}
$\overline{\text { Abstract }}$
Geographic Information Systems (GIS) are a proven resource for public transportation service planning and evaluation. In particular, their spatial analysis and database management capabilities make them well suited for such applications. The primary cost incurred in a GIS application is for the development and maintenance of high-quality spatial databases. Due to this cost, public transportation agencies want to utilize these databases to support a wider array of applications. As desktop GIS software and computer hardware become more powerful, GIS can be used to develop applications for "real-time" operations. In this research effort, a GIS-based prototype system was developed and tested to support the scheduling and dispatch functions of an on-demand flexroute transit service. The effectiveness of the prototype demonstrates the potential of GIS to support time-critical transit operations.
\end{abstract}

\section{Introduction}

GIS has proven to be a valuable resource for public transportation agencies. These agencies have applied GIS to a number of challenges, ranging from 
data management to service design. Although GIS provides numerous benefits, they are not realized without costs. Public transportation agencies have made significant investments in establishing and maintaining the spatial databases required by GIS. There is a great desire to take advantage of these investments in as many applications as possible.

In the past, GIS has been used almost exclusively for "off-line" planning and analysis applications in public transportation. However, recent advances in computer hardware and GIS software have now made the use of GIS in realtime operations feasible. Such applications may enable public transportation agencies to meet their goals of enhancing transportation services and capitalizing on spatial database investment.

Although real-time transit applications of GIS are conceptually feasible, there have been no rigorous analyses of such potential applications. In this research effort, the concept of using GIS to support the real-time analysis and data management needs of flexroute transit was examined by developing a prototype GIS-based scheduling and dispatching support tool. Flexroute transit, also referred to as "route deviation transit," is a hybrid of fixed-route transit and paratransit. In a flexroute system, fixed-route service is provided at a limited number of fixed stops, and slack time is built into the schedule between these stops to allow buses to pick up and drop off passengers on an on-demand basis. Scheduling trips for on-demand customers and dispatching vehicles to ondemand locations require sophisticated spatial analysis and substantial data management. Based on the positive results of the research, it is likely that GIS will play a key role in meeting real-time public transportation operations needs.

\section{State of the Practice: GIS and Public Transportation}

Public transportation providers have used GIS for nearly a decade. The main impetus for transit agencies to use GIS is to allow for the integration of data from a variety of sources to perform a number of planning analyses that were traditionally completed manually and were quite time consuming. GIS has allowed transit agencies to store, manage, display, manipulate, and analyze their spatial and attribute data efficiently. The power of GIS lies in its analytical 
capabilities that simultaneously consider spatial and attribute information. Today's desktop GIS software allows users to perform routine spatial analyses, and use complex relational database management concepts to address elaborate planning problems using standard personal computing hardware.

Traditionally, transit agencies have used GIS to statically (offline) manage real estate assets and perform traditional transit analyses such as ridership forecasts, service route planning, and demographic analyses (Schweiger 1991). Today, these traditional uses remain the foundation of most transit agencies' GIS efforts, and to a large extent, dictate the data needs of the agency.

The management of public transportation real estate within a GIS normally includes both fixed assets and land management. To demonstrate the size and scope of databases required for these activities, consider the management of fixed assets. The fixed assets for a transit agency include items such as the location, inventory, and condition of transit stations; fixed bus stops; bus stop signage; and even storage yards. Each of these features possesses a host of descriptive attributes. With fixed bus stops, for example, a GIS database stores, for each stop, its physical location (latitude and longitude), its position along the route, whether it is a transfer facility, if it has a shelter, if it has a bench, and the number of embarking and disembarking riders.

A recent survey conducted by the Urban Transportation Monitor revealed that a primary GIS application in public transportation is to support core transit planning analyses (1999). These analyses, which include ridership forecasting, service route planning, and demographic analyses, are fundamental planning practices of any transit agency. Although actual processes associated with each of these analyses have not changed dramatically with the introduction of GIS, the ability of the transit agency to perform a more in-depth, exhaustive analysis has greatly improved. That is, more frequent updates of data are available, the amount of data has increased, and a number of data sources/data types are now available that allow for nontraditional "what-if" analyses to be performed.

\section{Investments Required}

The implementation of a GIS for any size transit agency is complex and time consuming. The GIS is composed of essentially three components: hard- 
ware, software, and data. The hardware and software required can be obtained relatively quickly and, with few exceptions, can be used for multiple applications. The data, however, are often dependent on the specific application. It is the acquisition of data that is one of the most important and expensive steps in effectively applying GIS.

Estimates of the costs associated with the development of GIS databases that will perform off-line analyses range from 50 percent to 80 percent of the total cost of GIS implementation (Huxhold and Levinshohn 1995; Opiela 1993). Although these estimates include database design and maintenance, the acquisition of data comprises the largest portion of these costs. What is clear, especially when the survey results of the Urban Transportation Monitor are considered, is that transit agencies view their investment in GIS data as significant, and they feel this investment must be capitalized on as much as possible (1999).

Most of the operations processes required of a public transportation agency rely on the management and analysis of spatial and attribute data. For example, when monitoring the status of a bus fleet, a transit agency needs to know the locations of vehicles and their attribute characteristics. Further, when vehicles are determined to be behind schedule, network analysis is required to determine the best approach to rectifying the problem. Most of these functions, as well as the data required to support them, are available in modern desktop GIS packages and existing transit spatial databases. In the past, these tools have not been used to provide this functionality due to the immaturity of GIS software and the relatively slow processing speed of moderately priced computers. This forced many agencies to purchase proprietary, specialized software that required data in formats that were often incompatible with standard GIS packages. To capitalize on GIS investments, and to avoid compiling similar spatial databases in different, incompatible formats, many feel that the time has come for operational applications of GIS in transit. This article details a prototype system of this type.

\section{Prototype GIS-Based System}

Although the use of GIS to support real-time public transportation opera- 
tions is conceptually feasible, it is well known that the complexity of an application is not fully understood until it is developed. To explore real-time GIS applications fully, this research effort focused on developing a prototype GISbased support system for flexroute transit. Such a system must meet strict realtime requirements to support flexroute operations effectively and, therefore, provides an excellent test case for this effort. A full description of flexroute transit and a discussion of its challenges are presented below.

To provide further context for the research effort, the flexroute support system was designed and developed in cooperation with the Peninsula Transportation District Commission (Pentran), the public transportation provider in the Hampton/Newport News area of southeast Virginia. Pentran officials guided the development of the system to support flexroute service along two of its existing fixed routes that serve relatively low-density, suburban areas. Pentran believes this area holds high potential to support flexroute service, and is currently working to obtain funding to purchase the necessary equipment to implement the service on a trial basis.

An important aspect of working jointly with Pentran was that it allowed the development of the flexroute support system to be driven by functional requirements. A major change in the GIS application process required by realtime systems is to shift from an "experimental" approach to a software development approach. Off-line analysis applications typically require that an expert analyst work directly with the GIS in an experimental fashion attempting to derive information from the data through a series of queries and analytical processes. This approach is driven by the quest to derive information to support decision-making. Alternatively, real-time applications of GIS must be approached in a software development manner. The application is being developed to support a mission-critical operation. A rigorous set of software development techniques has evolved in the software engineering community to support the growth of applications that satisfy mission-critical requirements. These approaches, whether the classic waterfall or the newer rapid prototyping approaches, are based on a sound, thorough elicitation of the application's requirements (Eisner 1998). Pentran's involvement in this research allowed the 
team to develop the prototype application based on a full set of requirements.

The GIS software chosen as the foundation for this effort is ESRI's ArcView. ArcView was chosen for the following key reasons:

- It is an off-the-shelf package that is readily available.

- It can be customized through its internal programming language, Avenue.

- ESRI is an industry leader, and its spatial data format, the Shapefile, is publicly available. This ensures that the data required can be imported/exported from this application to other GIS applications.

\section{Flexroute Transit}

Flexroute service can be defined as "a service where the transit vehicle stops at fixed locations on predetermined schedules while also providing ondemand service to customers off the standard route between the fixed stops." As seen in Figure 1, the underlying network structure for flexroute service is different than that of fixed-route and paratransit services. The primary objective in designing a fixed-route service is to locate the fixed stops within a 0.25 mile walking distance of the surrounding population (Gray and Hoel 1992), whereas a flexroute service must be designed with the objectives of (1) serv-

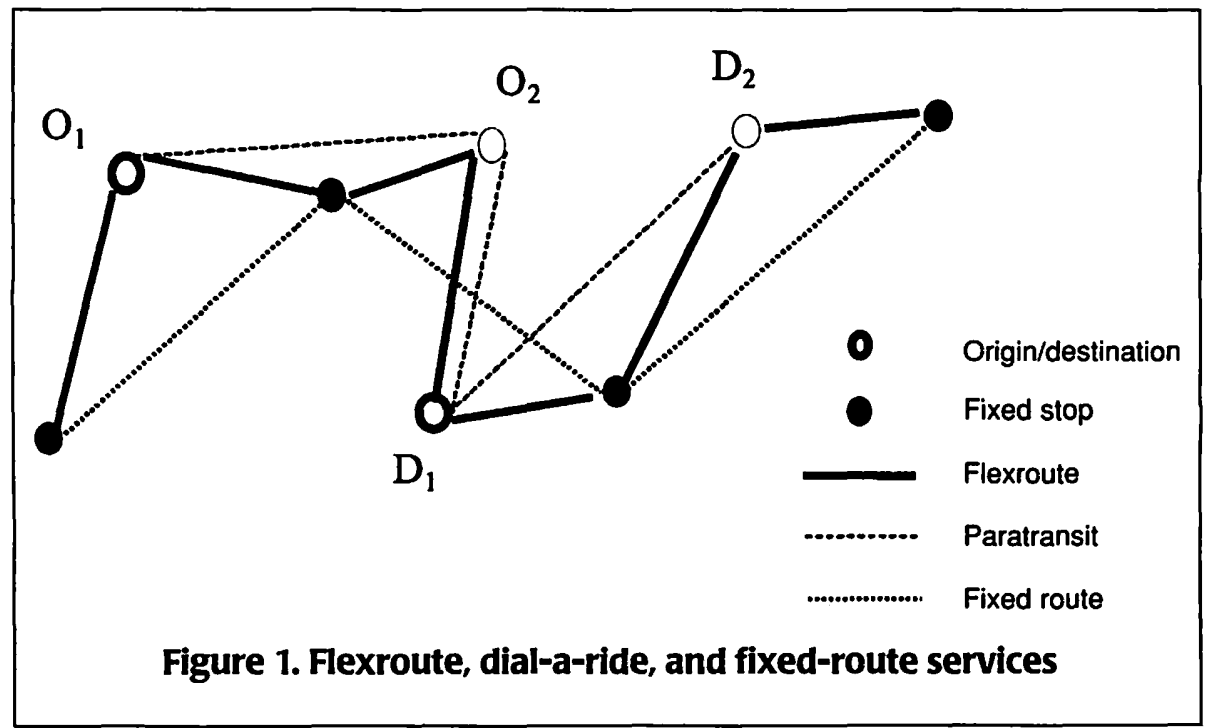


ing high-ridership fixed stops and (2) building the necessary slack time in the schedule to allow for deviated, on-demand pickups and deliveries. As in fixedroute service, the bus makes mandatory stops at the fixed stops and adheres to the constraint of arriving on schedule at these stops.

A service zone in a flexroute service is defined as the region between two consecutive fixed stops. The service zone defines how far away from the standard route a vehicle may deviate to pick up or drop off a passenger (i.e., the maximum deviation distance). It does not imply that everyone within the zone is guaranteed a deviated trip. At times, other committed rides will force a requested trip from within a service zone to be denied.

Two directions of travel are defined between the end points of the routes: inbound and outbound. These directions are global, and the definitions hold for all routes in a flexroute system. The bus is said to be traveling in the inbound direction if it is traveling in a general direction that is going toward the downtown area of the city, and the reverse is true for the outbound direction. For this study, the total end-to-end travel time for a route was one hour, along with onehour headways at each stop in each direction of the route. This design choice was dictated by a desire to maintain consistency with the rest of Pentran's fixed-route service.

The total end-to-end time includes slack time for deviation. A run or a pass of a bus is defined as "continuous travel of the bus from one end of the route to the other in one direction." Thus, in a time span of one hour, the bus on a route would have made one pass in one direction. This is an important definition specific to this problem and serves as a method to track customer pickups and deliveries for a given time period.

The scheduling and dispatching of a flexroute service in a real-time urban environment require certain essential system components (Round and Cervero 1996). First, there must be an automatic vehicle location (AVL) system so that a dispatcher has access to instantaneous information about the location of the vehicles in the system. Second, a spatial database is required to locate enroute vehicles on a map of the service area, store system-specific information, and view the street network. Third, there must be a communication system that can 
send information between the vehicles and the transit control center. Finally, a support system that is specifically designed for flexroute service is needed to process the real-time inputs from the subsystems, along with managing customer requests for service.

There are significant institutional, political, and technical issues to resolve before flexroute service will serve as a viable public transportation alternative. For example, it is unlikely that on-demand flexroute service can be provided on narrow residential streets using the current standard, 40-foot transit vehicles. These issues are real and substantive. However, they are of little significance if flexroute service is not feasible from a scheduling and dispatching perspective. Therefore, this research focuses on the challenge of flexroute scheduling and dispatch. Future efforts are required to investigate the equipment considerations involved in the implementation of flexroute transit.

\section{Flexroute Scheduling and Dispatching Problem Decomposition}

The routing and scheduling of a flexroute request in real time is a difficult task because of the combined characteristics of fixed-route and demandresponsive service. For instance, when a request for deviation is received, three important attributes must be determined before proceeding with the scheduling process:

1. The direction of travel for the trip request to determine the appropriate fixed-route vehicle for trip assignment. This is required because of the fixed-route component of flexroute service.

2. The spatial location of the pickup and delivery point relative to the fixed stops and the service area for flexroute.

3. The traveler's temporal requirements of the pickup and delivery points relative to the scheduled times of the fixed stops.

This spatio-temporal nature of flexroute service renders it different from the general paratransit scheduling problem. To solve it efficiently, the overall problem was decomposed into several subproblems. This was done by taking advantage of the unique service characteristics of the flexroute problem. Unlike paratransit services, the scheduled fixed stops in the route deviation service provide spatial and temporal anchor points on the route traversed by the 
transit vehicle. The transit vehicle must visit the fixed stops at the scheduled times amid picking up and dropping off on-demand riders.

In addition, on-demand service is provided only in the region between two successive fixed stops. This implies that each on-demand (as opposed to fixed-stop) pickup or delivery is restricted to the region between the fixed stops. This relationship between pickup and delivery points and service areas was used in the scheduling process. Further, each service zone on each route and direction is distinguished by a unique identification. The spatial and temporal location of the pickup and delivery points for a given trip request can be identified by determining the service zone it falls in. The functional steps involved in scheduling a flexroute trip are described below.

\section{Locate the Pickup and Dropoff Point}

Upon receipt of a call requesting flexroute service, the geographic location of the passenger's pickup and dropoff point relative to the service area must be determined. The spatial location should be identified given the street addresses of the two points.

\section{Find the Global Direction of Travel}

To assign the customer to a particular bus, first the global direction of travel for the request must be determined. This can be done by defining a fixed reference axis for the entire network. The reference axis is arbitrarily defined so that the axis passes through an origin point so that the entire service area falls in one quadrant (see Figure 2). The angles $\theta$ and $\phi$ are defined as:

$\theta=$ The angle between the reference axis and the line joining the pickup point and the origin of the axis.

$\phi=$ The angle between the reference axis and the line joining the delivery point and the origin of the axis.

Figure 2 clearly illustrates how the angles for the origin and destination (O-D) will be determined. The customer's global direction of travel can be determined from the following: 


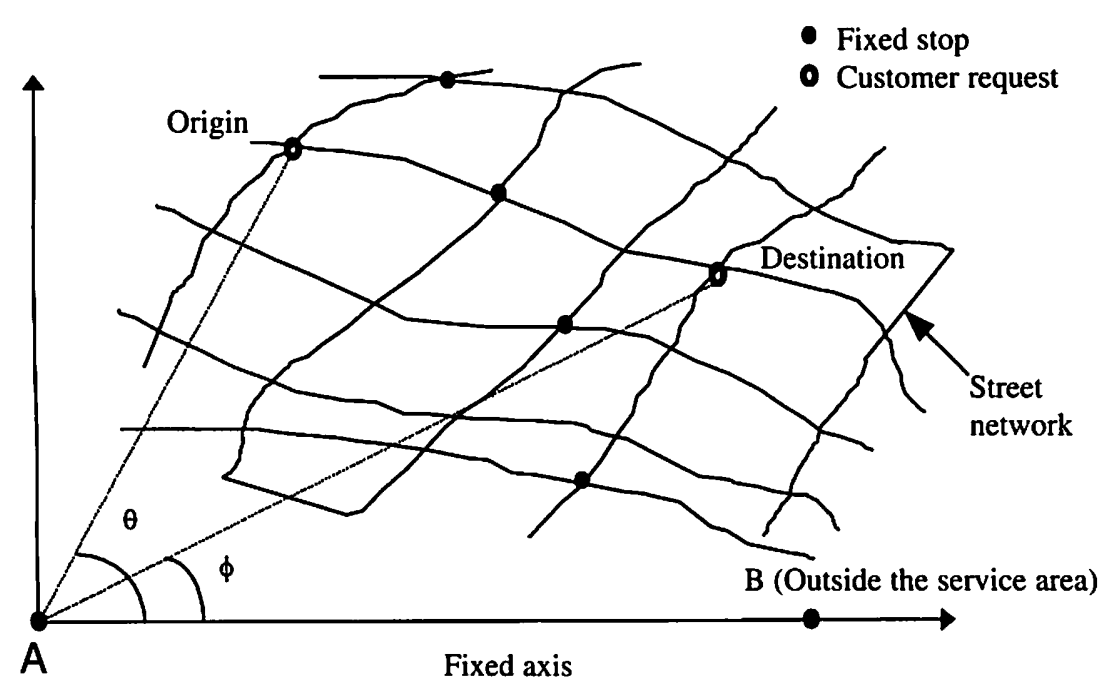

Figure 2. Finding the global direction of travel

Direction $=\left\{\begin{array}{l}\text { Inbound, if } \theta \geq \phi \\ \text { Outbound, if } \theta \leq \phi\end{array}\right.$

To determine the angles, it is necessary to know the coordinates of the origin $\left(\mathrm{x}^{o}, \mathrm{y}^{o}\right)$ and the destination $\left(\mathrm{x}^{d}, \mathrm{y}^{d}\right)$ points. The reference axis is a line passing between an arbitrary point $A$ and another point $B$ that is on the outer limit of the service area. Point $A$ will be assigned such that $y_{A}=y_{B}$ and $x_{A} \neq x_{B}$. The value of the angles can then be calculated by:

$$
\theta=\tan ^{-1}\left(\frac{y_{a}-y_{A}}{x_{a}-x_{A}}\right) \text { and } \phi=\tan ^{-1}\left(\frac{y_{d}-y_{A}}{x_{d}-x_{A}}\right)
$$




\section{Identify Eligibility for Service}

Once the spatial locations of the pickup and delivery points are found, the next step is to see if the passenger is eligible for flexroute service. The following criteria were used to determine eligibility for service:

1. The pickup and delivery points of a request should lie entirely within the designated service zones of a flexroute service.

2. Both the pickup and delivery points should not be within 0.25 mile of a fixed stop.

In other words, the proximity of the pickup and delivery points to the service zones and fixed stops must be determined to identify a passenger's eligibility for service.

\section{Store a List of Previously Committed Rides}

One of the important inputs to the scheduling process is the information on previously committed ride requests for a given service zone and pass of the bus. Therefore, information on the estimated time of pickup and delivery and their spatial locations must be stored for each previously committed ride for each service zone and bus pass combination. This information is needed to determine the feasibility of accepting the new ride request.

\section{Solve the Network Problem}

From the previous steps, important information about the spatial and temporal location of the request's pickup and delivery point is known. The problem now distills to determining whether a customer can be picked up and dropped off within the respective service zones without violating the time constraints at the fixed stops on either end of a zone as well as the time window constraints of previously committed riders in that zone. The answer to this problem can be obtained by finding the shortest path starting from one fixed stop, traversing through the intermediate pickup/dropoff points in that zone (including prescheduled and the current request under consideration), and ending at the subsequent fixed stop.

\section{Prototype GIS-Based Scheduling and Dispatch System}

To investigate the feasibility of using GIS as a foundation to meet the 
flexroute scheduling and dispatch requirements described above, a prototype GIS-based system was developed for the two Pentran routes. A description of how the capabilities of GIS were used to meet the requirements outlined in the previous section is provided below.

\section{Address Geocoding}

The address geocoding capabilities of GIS were used to meet the first requirement of determining the spatial location of the pickup and dropoff points for a trip request. Most desktop GIS software, such as ArcView, includes full geocoding capabilities. A street address is geocoded within a GIS by querying the table of street names with address ranges and using a scoring system to determine if a match has been found. This table is the attribute table for the street network layer. Once a match is found, the latitude/longitude coordinates of that street segment are assigned to the point and it is plotted on the map. The latitude/longitude coordinates of points within a GIS provided a convenient preexisting system for determining the angles $\theta$ and $\phi$ in the second requirement for finding the global direction of travel. The reference axis was created as a line feature on the map.

\section{Analysis of Spatial Relations}

Complex spatial relation analyses can be performed using most standard, off-the-shelf GIS software. For instance, in a spatial relation analysis, the user can select point features in one layer that are at a certain distance from the line features in another layer. This functionality, usually referred to as "buffering," allows GIS to assist in proximity analyses needed in applications such as flexroute scheduling and dispatching.

In this study, the developers created the individual service zones associated with each pair of consecutive fixed stops along each route as polygon features and stored them as a separate layer. Two such layers were created for each direction of travel. This was achieved by using the buffer feature of a standard GIS software, such as ArcView. A unique pair of fixed stops, the fixed route it belongs to, and the direction of travel identified each zone or polygon. The service zones in which the $\mathrm{O}-\mathrm{D}$ fell were determined by performing an "overlay" selection on the point layer containing the $\mathrm{O}-\mathrm{D}$ locations and the polygon layer 
with the service zones. Based on the selection, the system can immediately find the nearest pair of fixed stops for the O-D points of a request, depending on the polygon they fall within. In this step, the system also determines if there is a transfer involved in the trip by checking if the origin bus route and the destination bus route are the same.

Similarly, layers with point features were created to store the information describing previously committed rides for each zone, direction, route, pass, and day of the week, for the past one week. The nomenclature used to name each of these point layers was:

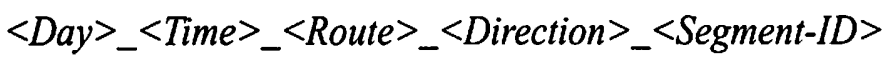

For example, the point layer with the name "MON_1500_11_IN_12" contains the scheduled requests for the most recent Monday, for the 3 P.M. pass on Route 11 in the inbound direction on zone 12. The attribute tables of these layers contain the information on the scheduled time of arrival or departure for each prescheduled on-demand stop. This information was used in the next step to check for violation of time windows. For each request, point layers for the O-D zones are obtained and passed on as inputs to the next step.

The layer chosen depends on the particular pass on the route in question. The pass is selected based on the passenger's preferred pickup or arrival times. In some cases, it may be necessary to analyze more than one pass to identify the itinerary that best suits the passenger's needs.

\section{Network Analysis}

Most desktop GIS software packages have basic network analysis capabilities, such as solving the shortest path between a set of points. For this research, the Network Analyst extension of ArcView was used to solve the shortest-path problem. Network Analyst can solve the following cases:

1. Find the shortest path given a set of points in the given order.

2. Find the shortest path through a set of points in the given order and returning to the origin.

3. Find the shortest path through a set of points by finding the best order of 
traversal and also returning to the origin. This option is similar to the traveling salesman problem. The best order is found exhaustively by computing the shortest path from each point to every other point.

Unfortunately, the shortest-path problem formulation required for this application does not fit one of these three categories. In this case, the path between two fixed stops and the intermediate on-demand stops could be addressed as a shortest-path problem with time window (SPPTW) constraints (due to the nature of the fixed stops). To implement this form of network search in ArcView, some modifications were made using the scripting language Avenue. The checks for the fixed-stop schedule violations and the time window constraints violations were performed by querying the attribute table of the resulting spatial layers created by the Network Analyst. Time window violations were checked only for the prescheduled requests for which the estimated pickup time (EPT) or estimated delivery time (EDT) were present. No time window checks were needed for the current pickup or delivery location. The total path cost found by the shortest path was then compared with the schedule of the fixed stop at the end of the segment to check for violation of schedule constraints. If no constraints are violated, then the trip is considered feasible and the order of traversal is stored. Here, the cost or time of arrival at the current location is noted and is then fixed as the EPT or EDT for the current location. The time window violation test used was:

$$
\begin{aligned}
& \text { EPT }-5 \text { (minutes) } \leq \text { (the new computed cost }) \leq \mathrm{EPT}+5 \text { (minutes), or } \\
& \text { EDT }-5 \text { (minutes) } \leq \text { (the new computed cost }) \leq \mathrm{EDT}+5 \text { (minutes) }
\end{aligned}
$$

Apart from the spatial layer showing the final path and the best order and cost of traversal of the points, the Network Analyst also stores and displays directions for the path. The results from this final step can be divided into four possible scenarios: (1) both the pickup and delivery of the request are feasible, (2) only the pickup is feasible, (3) only the delivery is feasible, and (4) both the pickup and delivery are not feasible. A request is considered "feasible" and is entered into the table of requests only for case (1). 
It is likely that as a flexroute transit service gains in popularity, the large number of committed rides will increase the difficulty of meeting both pickup and delivery requests while maintaining the schedule at fixed stops. In this event, the system provider may choose to modify the service to provide more slack time between fixed stops (to accommodate more deviations) or to decrease the headway between bus passes (to reduce the number of deviations per pass).

\section{System Performance}

The prototype system performed very well. On average, an operator could schedule a request for flexroute service within four minutes using a Pentiumclass personal computer. This time includes the customer interaction time required for the task (such as the time spent waiting for the customer to state his or her origin and destination). This meets the real-time requirements for such a service. It also demonstrates that desktop GIS software and "standard" desktop hardware are capable of supporting the complex spatial analyses and database management required for sophisticated real-time transit applications. Furthermore, the data used to support the application were derived from readily available spatial data currently used by transit agencies. This demonstrates the ability to realize a greater return on a transit agency's investments in spatial databases.

\section{Conclusions}

The prototype GIS-based support system reported here can be used as the foundation for developing full-fledged flexroute scheduling and dispatch software. Further, the results of this study demonstrate the applicability of GIS software for developing a customized decision-support system for real-time transit operations. The findings also demonstrate the capabilities of the GIS software in handling complex spatial and network analyses. With desktop GIS software becoming more and more powerful and easier to use, there are many advantages to storing and managing all of a transit agency's spatial and attribute data within a GIS database. Such a system could be used to support transit operations and conventional planning simultaneously, potentially eliminating the need to invest in separate, proprietary scheduling software. In addi- 
tion, with both the scheduling and planning systems in one place, any updates to the database can be made efficiently.

\section{Acknowledgment}

The research reported on in this article was funded by the Peninsula Transportation District Commission, the Virginia Transportation Research Council, and the Mid-Atlantic Universities Transportation Center.

\section{References}

Eisner, Howard. 1998. Essentials of project and systems engineering management. New York: John Wiley and Sons.

Gray, George E., and Lester A. Hoel (eds.). 1992. Public transportation. Englewood Cliffs, NJ: Prentice-Hall, Inc.

Huxhold, W. E., and A. G. Levinshohn. 1995. Managing Geographic Information Systems projects. New York: Oxford University Press.

Opiela, K. S. 1993. Management guide for implementation of Geographic Information Systems (GIS) in state DOTs. National Cooperative Highway Research Program Research Results Digest (191).

Round, Alfred, and Robert Cervero. 1996. Future ride: Adapting new technologies to paratransit in the United States. Working Paper UCTC No. 306, University of California Transportation Center, Berkeley.

Schweiger, C. L. 1991. Current use of Geographic Information Systems in transit planning. Washington, DC: U.S. Department of Transportation, DOT-T-92-02.

Urban Transportation Monitor. March 19, 1999. 13(5): 8-11.

\section{About the Authors}

BRIAN SMITH (briansmith@virginia.edu) is an assistant professor in the Department of Civil Engineering at the University of Virginia. Dr. Smith's research interests are Intelligent Transportation Systems, transportation systems management, and advanced information technology applications.

PriYa DuRvasula (priya@alk.com) is a software systems engineer at ALK Associates, Inc. She recently completed her doctorate in civil engineering at the University of Virginia. The research reported here was part of her doctoral work. Her research interests include GIS-T applications, freight rail transportation, and Intelligent Transportation Systems. 
STEPHEN BRICH (BrichSC@vadot.state.vaus) is a senior research scientist at the Virginia Transportation Research Council. His research interest areas include intermodal and multimodal transportation systems, traffic operations, and Geographic Information Systems. 


\section{Planning and Analyzing Transit Networks: An Integrated Approach Regarding Requirements of Passengers and Operators}

Markus Friedrich, Transport Planning Systems Unit, PTV

Thomas Haupt, Transportation Modeling Unit, PTV Klaus Noekel, Research and Development Unit, PTV

\section{$\overline{\text { Abstract }}$}

Providing an equally sufficient and efficient transit service requires careful planning and permanent monitoring of service quality, operating costs, and revenues. These requirements need a model that is capable of determining impacts on passengers as well as operators. Additionally, it is important to provide suitable and powerful methods to design and to modify the transit network. The transport planning software VISUM attempts to fulfill these requirements. In contrast to conventional Geographic Information Systems (GIS), which are extended to provide specific functionality for transit planning, VISUM is a comprehensive transportation model with additional GIS functionality. It seeks to fill the gap between conventional GIS programs and vehicle scheduling programs. 


\section{Introduction}

Demands for a competitive public transport that offers alternatives to private transport with minimal public subsidies call for a planning process that considers the impacts on passengers and operators. To passengers, good service quality means:

- short travel time,

- minimum number of transfers,

- good service frequency, and

- reasonable fares.

Operators and transit agencies must provide service in an economically efficient way. They need to monitor the performance of the existing service and forecast the impact of proposed measures. The operator, for example, needs to know the:

- required fleet size,

- operating costs,

- revenues from tickets, and

- cost coverage that indicates whether public subsidies are necessary.

These requirements of passengers and operators describe the fundamental conflict in transit planning. To solve this conflict, the transport planner needs to find an acceptable balance between two incompatible planning objectives: the maximization of service quality and the minimization of operational costs and public subsidies. For this complex planning task, transport planners started to apply software approximately three decades ago. Today, they can select from a variety of software tools for strategic planning, the most common of which are Comprehensive Transportation Models (CTMs) and GIS.

\section{CTMs versus GIS}

CTMs (e.g., Emme/2, Trips, TRANPLAN) were developed specifically for transport planning purposes. They connect travel demand data and supply data to determine traffic flows through the network. At the core of the models are an assignment procedure distributing travel demand onto the link network. In the beginning, most models focused only on private transport. Extensions to cover public transport often adapted modeling techniques for private transport. 
As a result, the models often tended to lack appropriate assignment procedures for public transport (i.e., timetable-based assignment procedures) and hardly considered aspects of transit operation.

GIS (e.g., MapInfo, ARC/INFO) is widely used for a broad range of purposes. Providing a user-friendly environment, it is applied to manage, analyze, and display geographical information by connecting database tables with geographical objects. Examples of GIS applications in the field of transportation include building and maintaining road databases and determining the accessibility of transit stops. Since standard GIS functionality does not cover specific transport aspects, GIS users all over the world are more or less successfully trying to adapt GIS according to their planning requirements. For instance, the TransCAD system is often used for transport planning purposes. TransCAD offers an alternative to CTM by combining GIS and transportation modeling capabilities.

\section{The VISUM Approach}

This article examines the transit features supported by the transport planning software VISUM (Friedrich 1998, 1999), which is part of the PTV VISION transportation software suite (PTV 1999). VISUM is a software program for planning and analyzing transportation networks (Figure 1). It provides specific functionality for public transport to help analyze and evaluate an existing or a proposed public transport service from the perspective of operators as well as passengers. By providing additional GIS functionality, VISUM fills the gap between conventional GIS, CTM programs, and vehicle and crew scheduling systems.

The development of VISUM continues to be strongly influenced by the needs of European transit authorities and operators. With the introduction of competition and the privatization of state-owned railway companies (Meyer 1997), many European transport companies were reorganized. Simultaneously, an increasing emphasis was placed on improving the attractiveness and efficiency of the service. Consequently, transportation planning software was expected to meet the following requirements:

- Multimodal transport model: Since the main challenger of transit operators is not the competing operator but private car transport, transport 


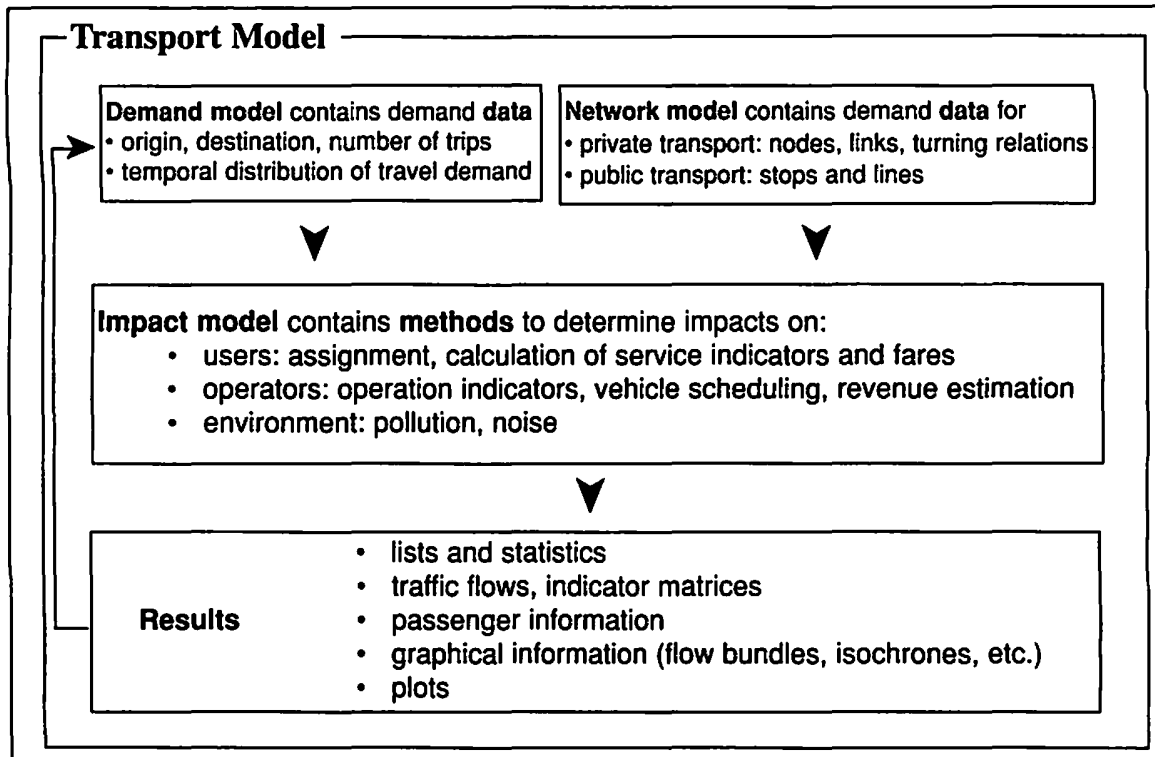

Figure 1. VISUM-comprehensive transport model and its submodels

models must integrate private and public supply systems in order to explore all the potentials for passengers.

- Continuous control: Transportation planning software is expected not only to assist during the planning process, but also to constantly monitor the performance of a transit system. Results of line-costing calculations serve as a continuous input for planning.

- Modeling of fare systems: Transparent and competitive fares are an essential prerequisite for success. This requires a model that allows the user to define fare zones and different types of tickets in order to estimate and optimize revenues. Transit networks with an integrated fare system depend on methods for distributing revenues onto lines of individual operators.

- Modeling of large networks: Railway companies and national transport planning agencies are in the process of establishing network models that incorporate precise line and timetable information on a nationwide or continent-wide level. 
VISUM attempts to cover these requirements. The specific transit features supported by VISUM include:

- Network model: VISUM offers a network model compatible with GIS as well as passenger information systems or vehicle and crew scheduling systems. As a result, it can combine geographical link network data and timetable data in an integrated network model.

- Fare model: VISUM provides a fare model to estimate revenues from ticket fares. The model supports distance-based as well as zone-based fares.

- Design process: VISUM provides functionality supporting the design process and assisting the planner in finding new solutions (e.g., by "drawing" the line-route on the screen).

- Service quality: VISUM includes specific assignment procedures for public transport that apply search algorithms similar to passenger information systems. This allows the user to examine the impacts on passengers by calculating essential service indicators and travel costs for each origin and destination (O-D) pair (journey time, waiting time, number of transfers, service frequency, fare).

- Line costing: VISUM supports line-costing calculations that state the profit or loss on individual transit lines regarding costs and revenues.

- Routes: VISUM offers the unique ability to store the routes of all passenger trips during assignment. Routes are most useful for postassignment analysis of traffic flows and the calculation of fares.

- Areas: VISUM can aggregate performance indicators as well as cost and revenue indicators for user-defined areas (e.g., traffic zones).

VISUM includes a demand model, VISEM, that estimates and forecasts mode-specific O-D matrices. The two basic features of VISEM are the classification of the population into behaviorally homogeneous population groups and the generation of trip chains derived from activity chains. For a more detailed description see Fellendorf et al. (1997). 


\section{Network Model}

The network model describes the supply side of the transport system consisting of several supply systems (Figure 2). Each supply system belongs to either the private transport (PrT) or public transport (PuT) mode, and uses one specific means of transport (car, truck, bike, bus, train, etc.). The combination of mode and means defines the system's characteristics determining a set of rules for the operation of the vehicles. The actual speed of individual transport vehicles is influenced by the network's capacity whereas transit vehicles operate according to their timetables. The requirements of an integrated network model for private and public transport influence the design of the network objects.

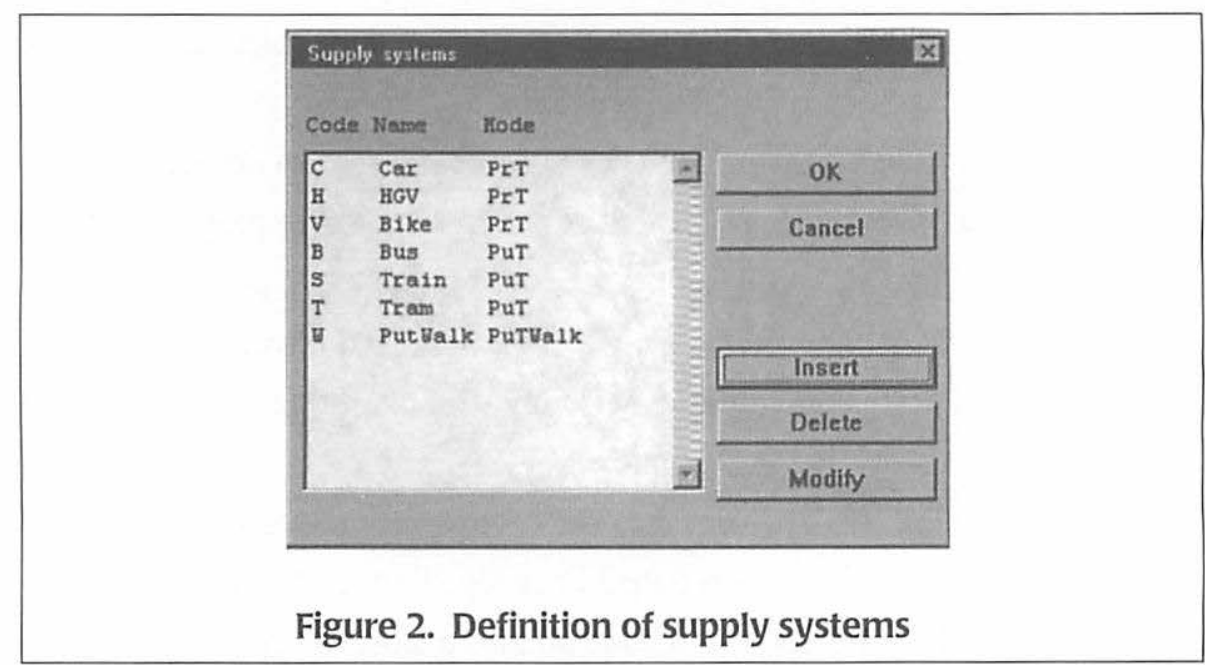

Source: Screen shot from VISUM 6.

- Nodes can represent intersections and/or public transport stops.

- Link attributes describe speed and capacity for private transport and carry default values for the running time of public transport vehicles.

- Turning relations penalize turning movements for private transport during assignment and define junctions for the construction of transit lines.

- Transit lines may use only links that are suitable for vehicles of the particular system. 


\section{Transit Lines}

A transit line has a particular line name and usually serves two directions. It may include one or several line variants (sublines) that show different lineroutes or running times between stops. A set of vehicle trips (service) defines the timetable, which can be calculated from the departure time of any vehicle trip at the origin stop and the running times between stops. A line belongs to one supply system and can, therefore, use only links that are permitted for this supply system (e.g., a bus may use only certain links of the road network while a train may use only the rail network). Each vehicle trip uses a defined type of vehicle that can carry information on vehicle-specific costs. Figure 3 shows a simple network with one bus line (Bus1). Figure 4 lists all relevant tables that are necessary to describe this network in a relational database.

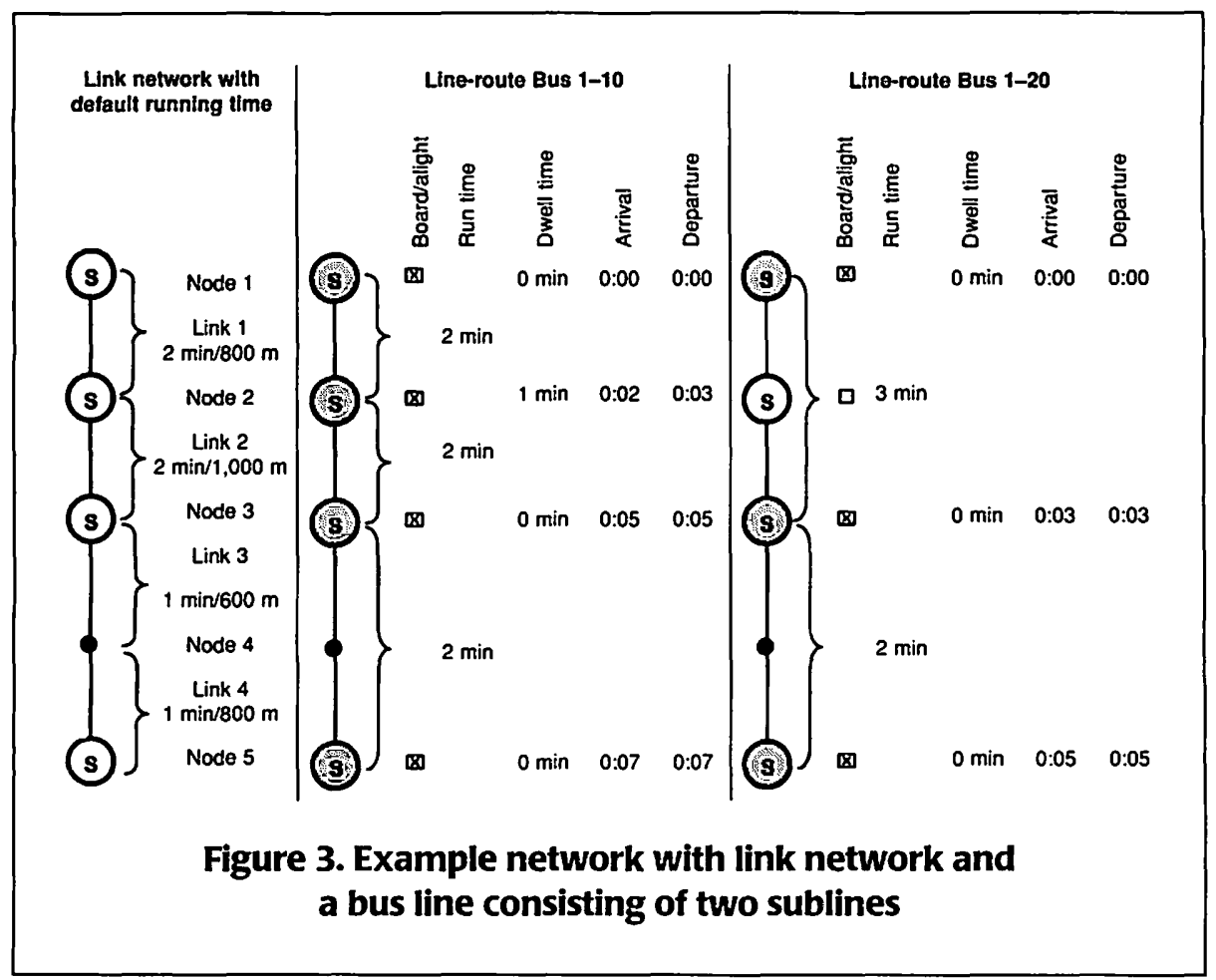




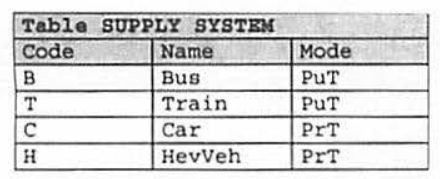

\begin{tabular}{|c|c|c|c|c|}
\hline NodeNo & Name & $\mathrm{x}$-Coord & $Y-\operatorname{coord}$ & Stop \\
\hline 1 & Node1 & $\ldots$ & & 図 \\
\hline 2 & Node2 & & & 图 \\
\hline 3 & Node 3 & & & 图 \\
\hline 4 & Node4 & & & $\square$ \\
\hline 5 & Node 5 & & & 図 \\
\hline
\end{tabular}

\begin{tabular}{|c|c|c|c|c|c|c|}
\hline 21 & & & & & & \\
\hline LinkNo & FromNode & ToNode & Length & Supplysystem & PrT-Capacity & RunTime (Bus) \\
\hline 1 & 1 & 2 & 800 & $B, C, H$ & $\ldots$ & $120 \mathrm{~s}$ \\
\hline 2 & 2 & 3 & 1000 & $\mathrm{~B}, \mathrm{C}, \mathrm{H}$ & & $120 \mathrm{~s}$ \\
\hline 3 & 3 & 4 & 600 & $B, C, H$ & & $60 \mathrm{~s}$ \\
\hline 4 & 4 & 5 & 8000 & $B, C, H$ & & $60 \mathrm{~s}$ \\
\hline
\end{tabular}

\begin{tabular}{|l|l|r|r|r|r|r|}
\hline Table VRHICLB \\
\hline VehTypeNr & Name & TotalCapacity & SeatCapacity & Cost/Hour & Cost/Km & Cost/Veh \\
\hline 1 & Midibus & 40 & 20 & 42,00 & 1,00 & 100 \\
\hline 2 & Standardbus & 90 & 35 & 42,00 & 1,50 & 150 \\
\hline
\end{tabular}

\begin{tabular}{|c|c|c|c|}
\hline Name & Variant & Direction & Supplysystem \\
\hline BUS1 & 1 & I (inbound) & $\mathrm{B}$ \\
\hline BUS1 & 1 & 0 (outbound) & $B$ \\
\hline BUS1 & 2 & I (inbound) & $B$ \\
\hline BUS1 & 2 & 0 (outbound) & $B$ \\
\hline
\end{tabular}

\begin{tabular}{|c|c|}
\hline \multicolumn{2}{|l|}{ Table OPBRATOR } \\
\hline OperatorNI & Name \\
\hline 1 & Urban Operator \\
\hline 2 & Railway Company \\
\hline
\end{tabular}

\begin{tabular}{|c|c|c|c|c|c|c|c|c|}
\hline Name & Variant & Direction & NodeNr & Board & Aliaht & Arrival & Departure & Length \\
\hline BUS1 & 1 & 0 & 1 & 圆 & 图 & $00: 00: 00$ & $00: 00: 00$ & 0 \\
\hline BUS1 & 1 & 0 & 2 & 国 & 图 & $00: 02: 00$ & $00: 03: 00$ & 800 \\
\hline BUS1 & 1 & 0 & 3 & 図 & 因 & $00: 05: 00$ & $00: 05: 00$ & 1000 \\
\hline BUS1 & 1 & 0 & 4 & 口 & $\square$ & $00: 00: 00$ & $00: 00: 00$ & 600 \\
\hline BUS1 & 1 & 0 & 5 & 图 & 図 & $00: 07: 00$ & $00: 07: 00$ & 800 \\
\hline BUS1 & 2 & 0 & 1 & 图 & 图 & $00: 00: 00$ & $00: 00: 00$ & 0 \\
\hline BUS1 & 2 & 0 & 2 & $\bar{\square}$ & $\square$ & $00: 00: 00$ & $00: 00: 00$ & 800 \\
\hline BUS1 & 2 & 0 & 3 & 国 & 図 & $00: 03: 00$ & $00: 03: 00$ & 1000 \\
\hline BUS1 & 2 & 은 & 4 & $\square$ & 므 & $00: 00: 00$ & $00: 00: 00$ & 600 \\
\hline BUS1 & 2 & 0 & 5 & 凶 & 図 & $00: 05: 00$ & $00: 05: 00$ & 800 \\
\hline
\end{tabular}

$\mid$\begin{tabular}{l|c|c|c|c|c|}
\hline Table TIMETABL \\
\hline Name & Variant & Direction & Departure & VehTypeNr & OperatorNr \\
\hline BUS1 & 1 & 0 & $6: 00: 00$ & 2 & 1 \\
\hline BUS1 & 1 & 0 & $6: 20: 00$ & 2 & 1 \\
\hline BUS1 & 1 & 0 & $6: 40: 00$ & 2 & 1 \\
\hline BUS1 & 2 & 0 & $6: 10: 00$ & 2 & 1 \\
\hline BUS1 & 2 & 0 & $6: 30: 00$ & 2 & 1 \\
\hline BUS1 & 2 & 0 & $6: 50: 00$ & 2 & 1 \\
\hline
\end{tabular}

Figure 4. Description of example network in a relational database 
- Table SUPPLY SYSTEM defines name and mode of each supply system.

- Table NODE contains the attributes of nodes that represent intersections or stops.

- Table LINK describes the link network. Each link is defined by two nodes and several input attributes (e.g., link length, permitted supply systems, free-flow speed, and capacity for private transport). The default running time for public transport systems is used during the interactive construction of a transit line in order to create a default timetable.

- Table VEHICLE defines types of transit vehicles. The capacity attributes allow the calculation of a line's saturation; the cost attributes are necessary to determine operating costs.

- Table SUBLINE defines lines and line variants.

- Table OPERATOR lists transit operators.

- Table LINE-ROUTE specifies a sequence of nodes and stops with running time between stops for each subline.

- Table TIMETABLE lists vehicle trips described by a departure time at the origin stop, a vehicle type, and an operator.

\section{Creating a Network Model}

Creating network models used to be a time-consuming task. The ability to obtain or purchase digital data opened new ways to build comprehensive and accurate network models. Main sources for multimodal network models are digital link network data (e.g., NavTech), which need to be connected with transit data containing information on transit stops, line-routes, and timetables. This involves a three-step process:

1. Import link network data using an interface to a GIS or relational database.

2. Import transit stops and match transit stops with nodes of the link network. The matching process geocodes the transit stops. Where transit stops do not find corresponding nodes, it is necessary to split links.

3. Import line-routes and timetables from the transit source. The transit source can be passenger information systems (e.g., Hafas, EFA), transit databases (e.g., Transmodel, UITP 1996), or vehicle scheduling systems 
(e.g., HASTUS, MICROBUS, INTERPLAN). Since these sources generally store only served stops with the line-route, the import process automatically inserts nodes into the line-route, to represent either intersections or through stops.

For importing and exporting network data, VISUM provides integrated interfaces to ASCII files (comma-separated values), relational databases (Microsoft Access, Oracle), and spreadsheets. Transit data can be accessed by product-specific interfaces or standardized transit databases.

\section{Interactive Construction of Transit Lines}

The main task of the planning process is the development of new solutions. Although new solutions may be generated through optimization algorithms, most solutions are still developed using the planner's creativity and experience, because the complex interdependencies within a transport system cannot be described appropriately through an objective function. Many practitioners spend a great amount of time modifying network data. On an extreme level, one can argue that as long as a planner prefers pen and paper for developing a first draft of a public transport network, the user interface of the modeling software needs to be improved.

In designing a transit line, a planner ideally wants to "draw" the lineroute on the screen. Operation aspects favor a line length that produces effective round-trip times and a stop sequence that ensures a sufficient catchment area. Passengers want fast, direct, and frequent line service with timed transfers.

The VISUM network editor provides a method to meet these requirements. In order to define a line-route, the user simply marks the two terminals of a transit line by a mouse click. Based on the link infrastructure, VISUM proposes a complete line-route with running times and distances. The proposed line-route may be subsequently modified by merely dragging parts of the line onto other links (Figure 5). Using a standardized timetable (e.g., peak hours/off-peak hours, 10-minute/20-minute headways) and an O-D matrix, it is possible to continuously inform the planner about the line's performance by displaying essential indicators in a status window: 


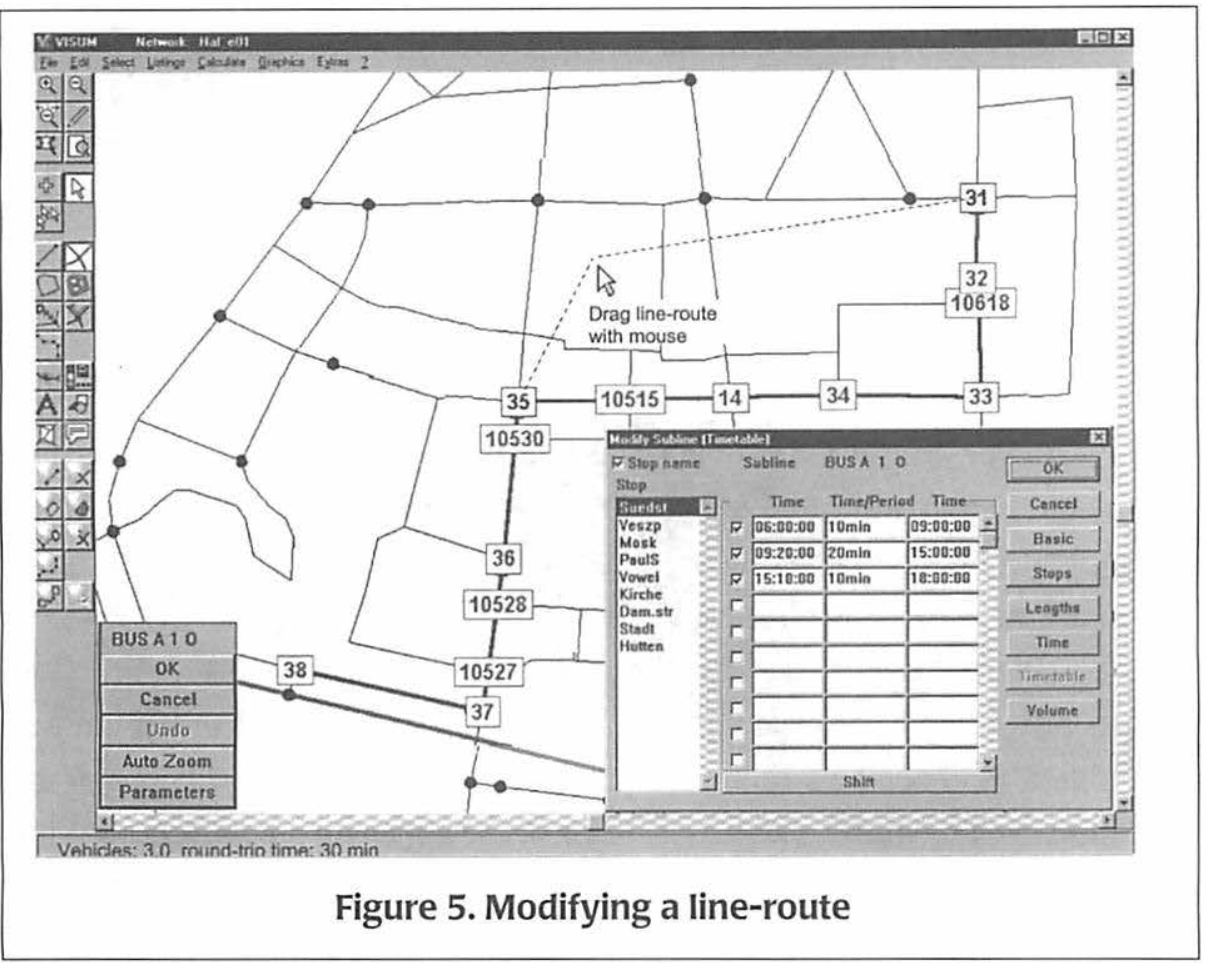

Source: Screen shot from VISUM 6.

- line length and running time,

- round-trip time and number of required vehicles, and

- O-D traffic that starts or ends within walking distance from the line's stops.

Advanced methods generate possible line-routes and optimize timetables with a fixed headway.

Line-Route Generation. This method (Sahling 1981) incorporates an objective function to minimize the number of transfers. Using a set of predefined terminals, it generates and evaluates a set of possible line-routes. The algorithm is based on an O-D matrix and the link network that may be used by lines. It considers existing lines and, therefore, allows the planner to focus on one transport system (e.g., bus) while other transport systems (e.g., train) are considered as fixed inputs. 
Timetable Optimization. This method (Guenther 1985, Maziejewski 1992) minimizes the transfer waiting time of passengers in a line network with a fixed headway. Based on the results of a public transport assignment, a genetic algorithm develops and evaluates "populations" of possible solutions by varying the departure time.

\section{User Model}

The objective of the user model is to determine the impacts of a transport supply system on travelers. Important indicators for evaluating the transport supply are journey time and travel expenses between two zones. To evaluate a public transport supply, additional indicators (e.g., number of transfers, transfer wait time, and service frequency) must be considered (Friedrich 1994).

To determine these service indicators, the passenger journeys are modeled. A private transport user chooses a convenient route for his or her journey. In addition to choosing a route, the public transport user also selects a departure time from the timetable; that is, he or she searches for a connection. While a route describes only the spatial course of a trip within a network, a connection additionally encompasses temporal constraints such as departure and arrival times at the origin stop, transfer stops, and the destination stop.

Methods to model the travel behavior are based on search algorithms that determine routes or connections between an O-D. So-called shortest path algorithms are used as search algorithms to determine the "best" route, that is, the one with the lowest impedance. Impedance can consist of times, distances, comfort restraints, and costs. Depending on the search algorithm used, the shortest path represents a route or connection. Based on the service indicators of each route/connection, the assignment then distributes the trips of an O-D pair onto the found routes or connections. As the characteristics of urban public transport and regional or interregional public transport differ, VISUM provides two special assignment procedures (Figure 6).

\section{Assignment Based on Lines}

The assignment procedure based on lines (assignment based on average headway) models each line through a sequence of stops, through the running times between the stops, and through the headway of the line. Lines with no 


\begin{tabular}{|c|c|}
\hline Assignment Based on Lines & Assignment Based on Timetables \\
\hline 1. Route Search & 1. Connection Search \\
\hline $\begin{array}{l}\text { Search for best route: } \\
\text { Impedance = } \\
\text { access time + egress time + in-vehicle time } \\
\quad+\text { transfer penalty } P \times \text { no. of transfers } \\
\quad+\text { mean transfer time ( }=\text { Fac } \times \text { mean headway) } \\
\text { Repeat search with different penalties } P \text { and } \\
\text { weightings of Fac to determine several routes }\end{array}$ & $\begin{array}{l}\text { Search for best connection: } \\
\text { Impedance }= \\
\quad \text { access time + egress time + in-vehicle time } \\
\quad+\text { transfer penalty } P \times \text { no. of transfers } \\
\quad+\text { actual transfer time } \\
\text { Repeat search for all possible departure times at } \\
\text { origin stop }\end{array}$ \\
\hline 2. Route Choice & 2. Connection Choice \\
\hline $\begin{array}{l}\text { Delete unattractive routes, where: } \\
\text { journey time }>\text { min. journey time } \times \text { factor } \\
\text { transfers }>\text { min. transfers }+ \text { factor }\end{array}$ & $\begin{array}{l}\text { Delete unattractive routes, where: } \\
\begin{aligned} & \text { journey time }>\text { min. journey time } \times \text { factor } \\
& \text { transfers }>\text { min. transfers + factor }\end{aligned}\end{array}$ \\
\hline 3. Route Split & 3. Connection Split \\
\hline $\begin{array}{l}\text { For each route calculate: } \\
\text { - perceived journey time PJT, which consists of } \\
\text { weighted components of journey time } \\
\text { - fare } \\
\text { - Impedance Imp =f (PFT, Fare) }\end{array}$ & $\begin{array}{l}\text { For each connection calculate: } \\
\text { - perceived journey time PJT, which consists of } \\
\text { weighted components of journey time } \\
\text { - fare } \\
\text { - temporal utility } U \text {, which results from } \\
\text { comparing the desired departure time of } \\
\text { passengers with the actual departure times of } \\
\text { the connection } \\
\text { - Impedance Imp = f (PFT, Fare, U) }\end{array}$ \\
\hline $\begin{array}{l}\text { Distribute trips with Kirchhoff Law: } \\
\qquad P_{i}=\frac{I m p_{i}^{-\alpha}}{\sum_{j=1}^{n} I m p_{j}^{-\alpha}}\end{array}$ & $\begin{array}{l}\text { proportion of trips using route/connection } i \\
\text { number of routes/connections } \\
\text { impedance of route/connection } i \\
\text { impedance sensitivity factor }\end{array}$ \\
\hline \multicolumn{2}{|c|}{$\begin{array}{c}\text { Figure 6. Characteristics of assignment based on lines } \\
\text { and assignment based on timetables }\end{array}$} \\
\hline
\end{tabular}

fixed-rhythm headway are described by their mean headway. This procedure does not explicitly calculate a transfer time but assumes that the transfer time depends on the headway. This means the coordination of the timetable is not considered. Usually the wait times at the boarding or transfer stops are equal to half of the line's headway. Assignment based on lines guarantees good assignment results for urban areas with a dense network and short headways.

\section{Assignment Based on Timetable}

The assignment procedure based on timetable (real-time assignment) con- 
siders the timetable of each transit line with its exact departure and arrival times (Friedrich 1994). A shortest-path algorithm based on these data calculates the "best" connection between two traffic zones for a particular departure time. For different times of departure, different "best" connections may be calculated that can differ by the used transit lines and/or transfer stops. To determine all "best" connections, the shortest-path algorithm is performed several times for each possible departure time within the assignment time interval. Passengers select from this set of possible connections. Their choice is influenced by the service indicators of each connection and by the utility of the departure time. The individual components of disutility (e.g., access time, transfer time, in-vehicle time, fare) are weighted with user-definable perceived unit costs. Assignment based on timetable is the appropriate method for rural areas or rail networks, where headways are long and the coordination of the timetable is important for the service quality. The exact calculation of connections, however, requires more computing time than the assignment based on lines.

\section{Assignment Results}

The assignment produces three types of results (Figure 7): traffic volumes on links, lines, and stops; service indictors for all O-D pairs; and routes. VISUM's unique feature of storing all routes during assignment allows extensive postassignment analysis of traffic flows. It can also be used to calculate revenues from passenger fares without performing a new assignment. Thus, it is possible to easily evaluate the impacts of new fare systems or higher fares concerning the revenue and cost coverage of lines.

\section{Operator Model}

To estimate the impacts on transit operators, the operator model is applied to determine indicators that express the operational and financial requirements for providing a transit service. The operator model supports line-costing calculations-a most useful tool for those responsible for strategic, financial, and operation planning. Line costing states the profit or loss on individual transit lines regarding costs and revenues.

In order to evaluate the performance of a transit line, it is necessary to deter- 


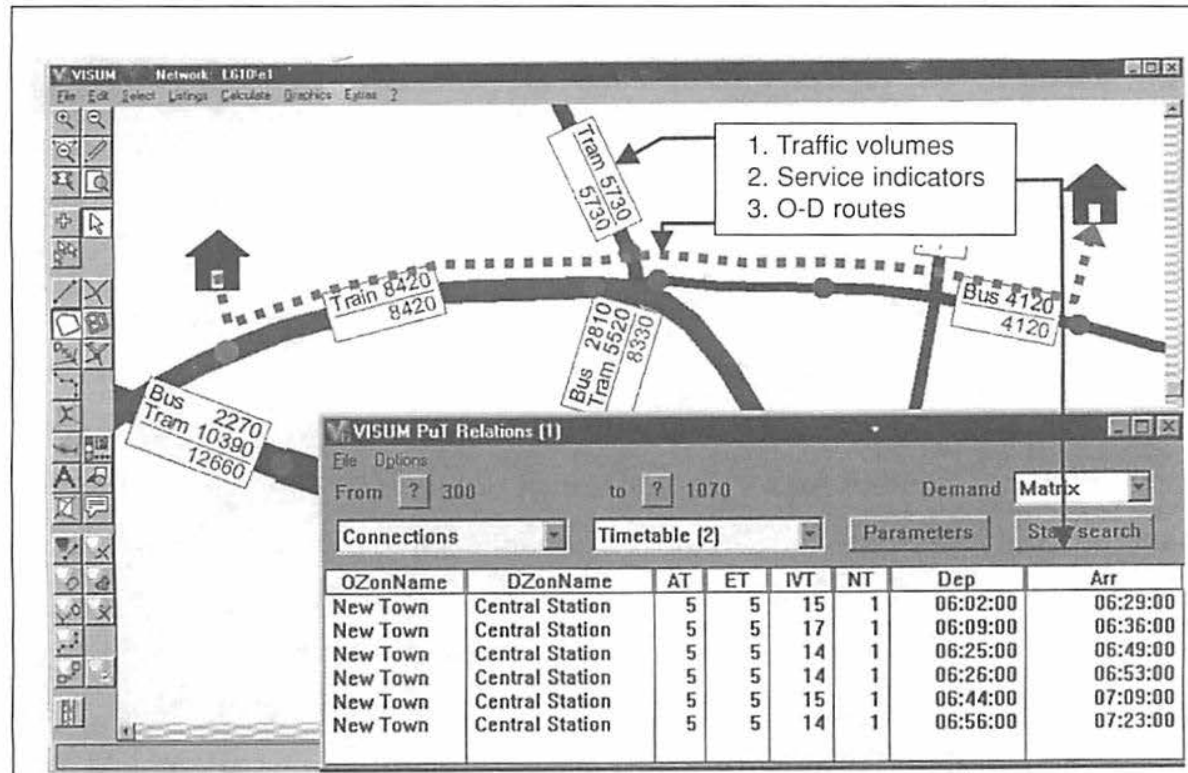

Figure 7. Assignment results: traffic volumes, service indicators, and routes

Source: Screen shot from VISUM 6.

mine indicators on a line level. This is easy for indicators such as vehicle kilometer, which can be directly calculated from the line length and the timetable. Indicators such as operating cost from vehicle depreciation or revenues from passenger fares, however, are more complicated, since a vehicle may be employed for several lines and a passenger may use more than one line for his or her journey. Operation indicators can be divided into the following categories:

- system performance indicators,

- vehicle requirement indicators,

- transport performance indicators,

- cost indicators, and

- revenue indicators.

\section{System Performance Indicators}

System performance indicators express operation requirements in kilometers or time units. They are calculated automatically after every modifica- 
tion of line data and do not require demand data. Examples of performance indicators are:

- Vehicle kilometer: Vehicle trip length $x$ number of vehicle trips.

- Service time: Time for passengers transport = line running time $x$ number of vehicle trips.

- Seat kilometer: Vehicle kilometer $x$ seats of vehicle.

\section{Vehicle Requirement Indicators}

VISUM provides an algorithm with which planners can estimate the number of required vehicles for a specified transit supply. The main goal of this calculation is to assign the total number of vehicle trips of an operational day to vehicles so that a minimum number of vehicles is required. The basis for this calculation is the timetable. It consists of individual vehicle trips that are described by subline, direction, and departure time from the first stop of the line. Vehicle rotation results from the concatenation of individual vehicle trips to trip chains that can each be performed by one vehicle. In the simplest case, a vehicle trip is concatenated at its last stop with a subsequent vehicle trip that starts at the same stop. If such a concatenation is not possible or useful, the vehicle can be redeployed to a different stop.

\section{Transport Performance Indicators}

Combining supply data with travel-demand data quantifies the transport performance described by indictors like number of boarding passengers, saturation, and passenger kilometer. These indicators are calculated automatically during assignment.

\section{Cost Indicators}

The costs of a line consist of these cost segments:

- Hourly costs: Time-dependent costs for personnel.

- Kilometer costs: Kilometer-dependent costs for fuel, repair, etc.

- Vehicle costs: Fixed costs for each required vehicle (depreciation, insurance).

- Network infrastructure costs: Costs from depreciation of new links or running costs for maintaining the network.

- Operator costs: Share of costs for overhead costs. 
Costs for vehicles, network infrastructure, or overhead need to be distributed to individual lines or vehicle trips. This requires a distribution key considering vehicle kilometer, seat kilometer, and service time.

\section{Revenue Indicators}

To estimate revenue from ticket fares, a revenue value per transported passenger is calculated considering the fare system (distance-based fare, zonebased fare). This revenue value is then distributed over the lines used by the passenger for one passenger trip. Revenue can be distributed onto the sections of a trip using the length of each trip section or the number of trip sections. Figure 8 shows an example with three different approaches to distribute a revenue of 3.00 monetary units onto two lines. Cost coverage of a line is calculated by comparing revenues and costs.

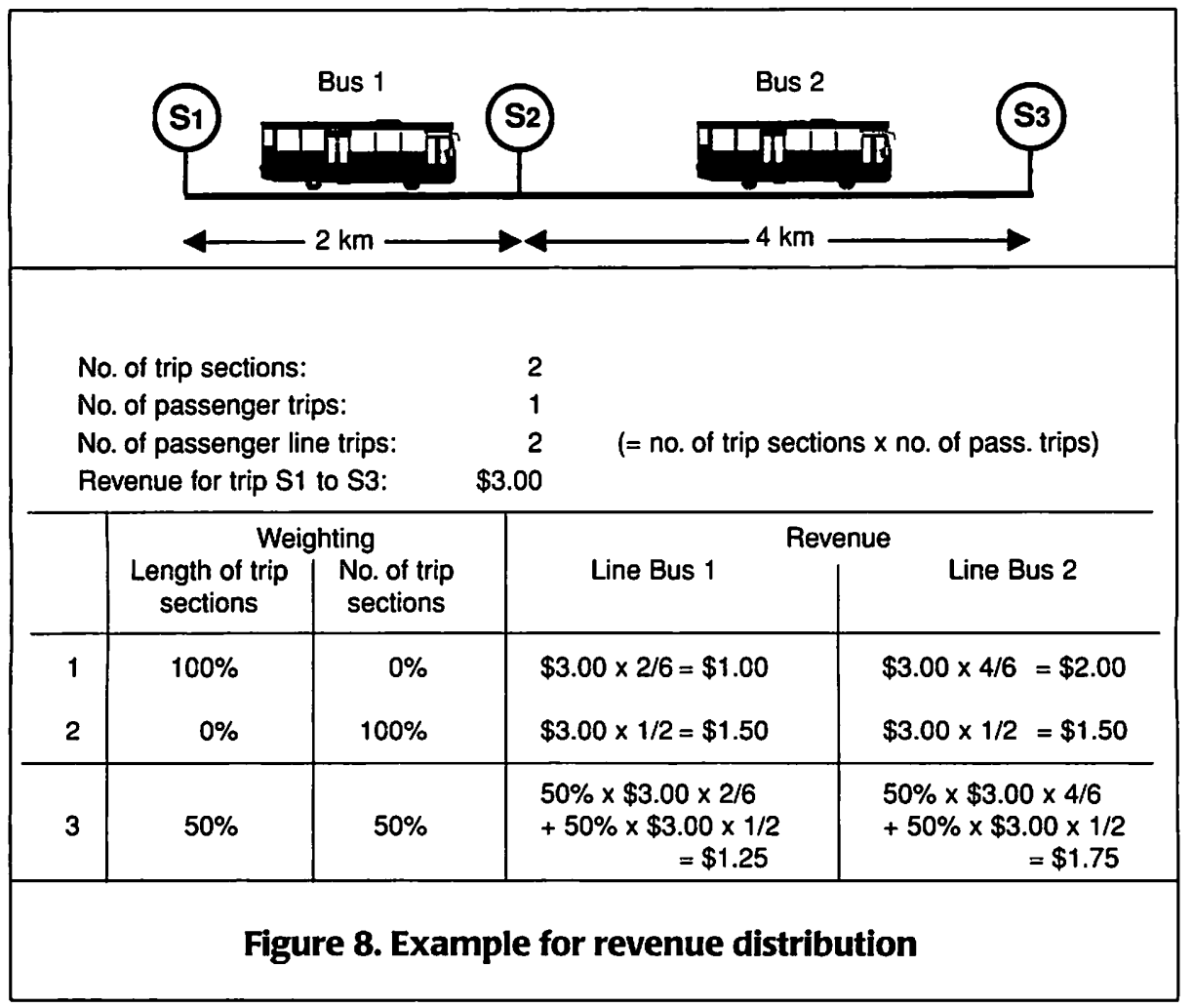




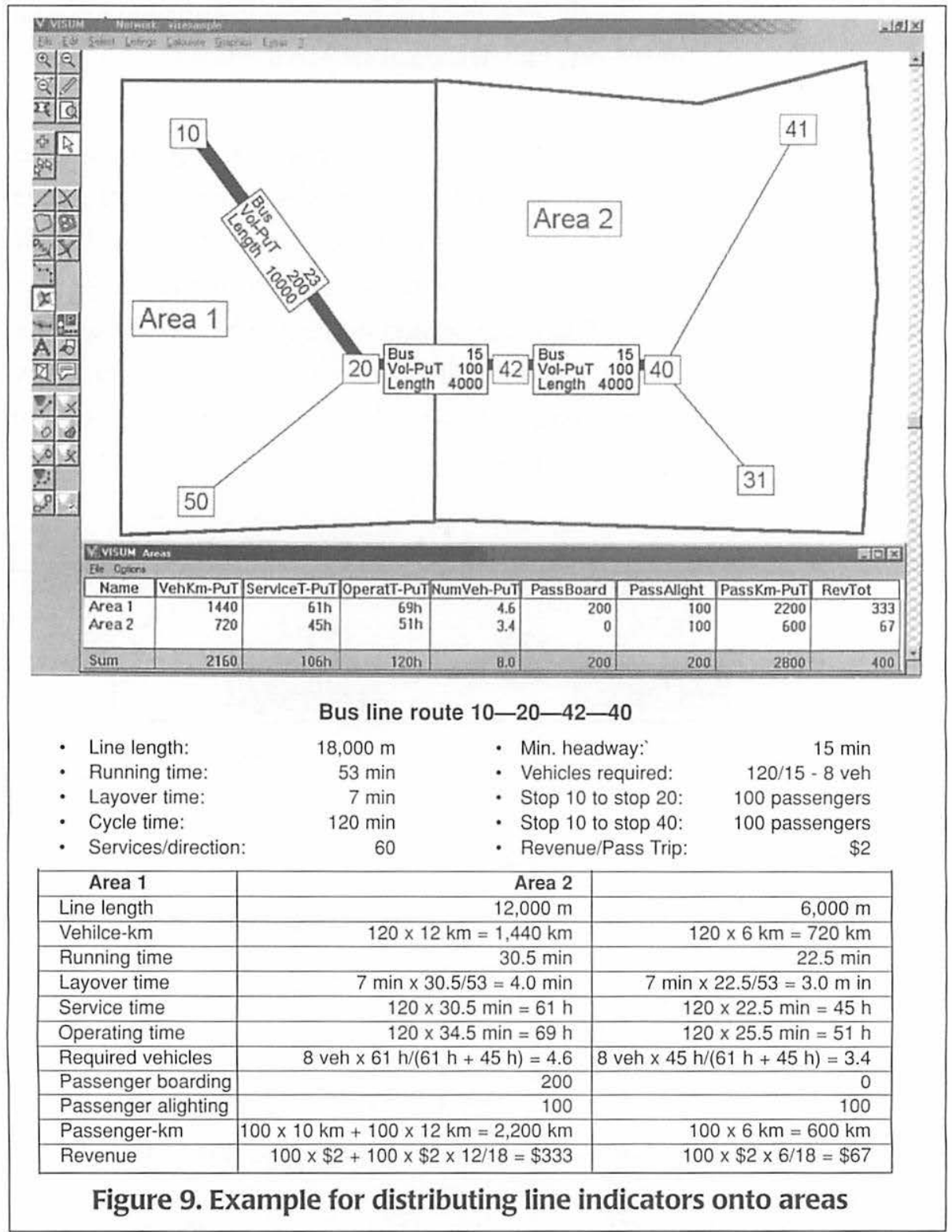

Source: Screen shot from VISUM 6. 


\section{Selected Analysis}

All performance indicators as well as cost and revenue indicators may be aggregated and displayed on the level of individual lines, supply systems, or areas (e.g., districts). In order to determine indicators for an area it is necessary to define a specific calculation routine for each indictor (Figure 9). Vehicle kilometers of a line can be distributed to areas proportional with the line length inside the area. This is not possible for the revenues of a line because a line might earn different revenues in each area. Therefore, the procedure for distributing revenues to areas must process each single passenger route with its individual revenues.

\section{Conclusions}

Demands for an efficient public transport network that offers alternatives to private transport and requires minimum public subsidies call for a planning process in which the impacts on passengers and operators are considered simultaneously. This requires a planning system that integrates:

- a disaggregated version of the four-step procedure (CTM),

- detailed transit data and specific methods to analyze the impacts of transit supply systems, and

- GIS capabilities for editing networks and analyzing spatial impacts.

VISUM combines these requirements, thus stimulating the planner within the planning process to experiment with alternative solutions. Various performance indicators are calculated concurrently with the modification of network data, so that the impacts of measures can be assessed easily. Since modifications of the transit supply directly influence operating costs and revenues, it is recommended that a line-costing calculation be included. Combining assignment results with a fare model allows measures like new fare systems and higher fares to be evaluated.

VISUM currently has almost 400 installations, mainly in Europe. VISUM is used for transit planning by cities (e.g., Berlin, Vienna), transit agencies (e.g., Dresden, VSN Group), and railway companies (e.g., DB AG, SNCF). Compared to other CTMs and GIS, VISUM supports a broad and detailed tran- 
sit function. (For a comparison of Emme/2 and VISUM, see SAMPLAN 1999.) Embedded in a multimodal model, its transit functions serve as powerful tools for planners involved in transit planning as well as integrated planning.

\section{References}

Fellendorf, M., T. Haupt, U. Heidel, and W. Scher. 1997. PTVVISION: Activity-based demand forecasting in daily practice. In Activity-based approaches to travel analysis. Oxford: Elsevier, 55-72.

Friedrich, M. 1994. Computer assisted design of public transport systems in rural areas. Schriftenreihe des Lehrsstuhls für Verkehrs-und Stadtplanung 5.

Friedrich, M. 1998. A multimodal transport model for integrated planning. Book of abstracts of the 8th World Conference on Transport Research.

Friedrich, M. 1999. Modeling public transport-A European approach, on Preprint CD-ROM of the 78th Annual Meeting. Transport Research Board.

Guenther, R. 1985. Planning and operational measures to improve connections in urban bus network. Schriftenreihe des Instituts für Verkehrsplanung und Verkehrswegebau 15.

Maziejewski, S. 1992. The vehicle routing and scheduling problem with time window constraints using genetic algorithms. Diploma thesis, Institut für Logistik, Komplexität, und Deduktionssysteme, Technical University of Karlsruhe.

Meyer, K. J. 1997. Regionalization and competition in Europe. Der nahverkehr 5/97. PTV Planung Transport Verkehr AG. 1999. www.ptv.de.

Sahling, B. M. 1981. Planning public transport lines. Institutsnotiz des Instituts für Verkehrswesen (29).

SAMPLAN. 1999. Choice of network analysis system for the new Swedish national model system SAMPERS. Swedish Institute for Transport and Communications Analysis.

UITP Union des Transports Public. 1996. Étude sur la normalisation des modèles de donnés en transport public. International Union of Public Transport.

\section{About the Authors}

MARKUS FrIEDRICH (markus.friedrich@ptv.de) directs the Transport Planning Systems Unit at PTV in Karlsruhe, Germany. He received his doctorate degree in transportation engineering from Munich University in 1994. 
Thomas Haupt (thomas.haupt@ptv.de) is director of the Business Transportation Modeling Unit at PTV.

KLAUS NOEKEL (klaus.noekel@ptv.de) is responsible for research and development at PTV. He received his doctorate degree in computer science from the University of Kaiserslautern, Germany, in 1990. 


\title{
Transit GIS Applications in Fairfax County, Virginia
}

\author{
Wenyu Jia and Brendan Ford \\ Fairfax County Geographic Information Services
}

\begin{abstract}
$\overline{\text { Abstract }}$
The Fairfax County Department of Transportation (DOT) manages a fixed-route bus system (the Fairfax Connector) that encompasses 58 routes. To better support the planning, operation, and marketing of this bus system, the Fairfax County DOT and the Fairfax County Department of Information Technology formed a team to develop a pilot project of Geographic Information Systems (GIS) transit applications. These applications would serve as a demonstration to facilitate automation, analysis, accessing, and plotting of transit data. To be successful, the applications had to be cost effective and match users' technical needs with their abilities.

Paramount to the success of this project was having a transit database capable of supporting all the applications identified by the development team. The database had to handle problems such as bus stops serving more than one route and routing varying by time of day. To effectively model the bus routes in GIS, each path had to be uniquely identified even though the same route could travel on different paths. Dynamic segmentation of ARC/INFO v.7.l was used to develop the route database. A bus stop database, in the format of ARC/INFO coverage, was then related to the route coverage.

More than 15 applications were identified for three areas of transit management: planning, operation, and marketing. Planning applications focused on transit service
\end{abstract}


improvement and route restructuring. They included routing adjustment, route demographic and land-use analysis, and reporting of statistics required by the National Transit Database. Operation applications were designed for daily service monitoring and consisted of route running times, loading at bus stops, and emergency service. Marketing applications emphasized functions for public outreach, which included creating specialized route and stop maps and publishing route information on the Internet. Most end-user applications were built in Arcliew 3.0 using Avenue and Dialog Designer. Some applications were built with ESRI's Map Objects (MO) and Map Objects Internet Map Server.

\section{Introduction}

Fairfax County is a fast-growing county in the Washington, D.C. metropolitan area. Since 1990, its population has maintained a steady growth of 2 percent from 0.8 million to 0.95 million in 1999. In the same time period, major commercial and industrial developments have occurred in the county. In the past two years, employment in Fairfax County grew at an annual pace of 5.7 percent. Growth was particularly strong in the telecommunication and information technology sectors. Growth and development coupled with aggravating traffic congestion on major corridors further pressed the need for transit service improvements.

The Transit Operations Division of the County's DOT is responsible for planning and marketing the service, and managing private operators. The Fairfax Connector carries an annual ridership of more than 4.7 million passengers. With major system expansion in July 1999, there should be significant ridership growth by the year 2000 . The issue of effective planning and efficient management, therefore, is critical.

GIS has been applied in Fairfax County's transit operations primarily for mapping needs. But it was not intended to facilitate routine tasks in a systematic approach. In May 1998, DOT introduced GIS in transit management. Input was first solicited from staff in the Transit Operations Division, and then a review was conducted between DOT staff and the County's Department of Information Technology (DIT) staff. During the process, development of a prototype for performing transit tasks through desktop GIS was desired. A list of 
selected applications was finalized for the transit GIS pilot project. In addition, a project team was formed of staff from both DOT and DIT. The team defined the pilot area: three routes and their associated stops in the southern portion of the county. These routes represent three different levels of services, including the most complex route in the system.

The objective of this project is to demonstrate the potential GIS would contribute to transit planning, marketing, and operations in Fairfax County. Therefore, it is designed to cover all three areas of interests. Planning applications address the analysis of transit service area; operation applications facilitate service monitoring; and marketing applications concentrate on public access to transit information as well as customized mapping. This article presents the results of the pilot project and discusses issues concerning systematic implementation that will follow the pilot phase.

\section{Data and Software}

The project started with the identification of existing data sources in the County in order to minimize raw data collection efforts at the initial phase. Street centerline data, already digitized by DIT, are an excellent base for route building. They offer a much higher level of accuracy than U.S. census Tiger files or any other commercially available data. They were originally produced in 1995 from 1-inch to 4,000-feet base maps. Land-use coverage data, important for planning analysis, were not readily available at this stage; therefore, a combination of the County's public facility data, planimetric data, and digital orthophotography was used to identify land-use features in the service area. Census block-group data provided a fairly detailed level of analysis. Several transit data sources are used in the applications, including transit ridership and boarding and alighting activities. The bus stop database developed by the DOT was used to create a geographic data layer of bus stops in ARC/INFO.

The digital orthophotography layer has proven extremely useful with transit applications as well as other GIS applications. The County GIS department purchased the photography in 1997 and has made it available to both County users and the public. It is viewable at an 8-foot pixel resolution on the Internet at www.co.fairfax.va.us/maps/maps.htm. The photography is served 
through a viewer (MrSid from LizardTech, Inc.). At the time of this writing, the GIS department was investigating solutions for yearly updates to this photography. Even with County personnel providing the ground control, it cost about $\$ 1,200$ per square mile. This price tag prohibits the County from updating the entire area every year. One solution is to update areas of high development each year based on available funds. Another is to update one-fourth of the county each year providing a four-year cycle of updates. Whatever the solution, the photography comes at no cost to user agencies such as transportation.

Also paramount to the success of most transit GIS projects is an up-todate street centerline layer. The street centerline layer is maintained in the GIS department and is updated as part of the GIS parcel maintenance application. Information is also provided by members of the Fire and Rescue Department and used to update the street centerline layer. As with the digital orthophotography, this information is stored on a central server and made available to all County agencies.

ARC/INFO was used to develop the route layer and the bus stop layer, both of which provide the base for the entire pilot project. Distance measures were acquired for both route and stop layers, which were later used in various applications. The development of route and stop layers is discussed in detail later in this article.

ArcView, due to its Windows-based desktop characteristics, is being used for the development of final applications for end-users.

\section{Building Routes and Stops}

As is often the case with suburban bus systems, one bus route can contain multiple paths, usually depending on the time of day, to serve varying demand. Modeling this situation in GIS requires that either each path (or route) is stored independently or that the bus route is broken down into its functional components and stored through a series of related tables. The project team contacted fellow GIS professionals with experience using the dynamic segmentation functionality in ARC/INFO. One of the most important points to consider was whether bus stops occur in the middle of street segments or at street intersections. With the former, ARC/INFO dynamic segmentation becomes much more 
a necessity. Another critical factor was the size of the route system and the degree of change. At least one site with a large route system had data stored in dynamic segmentation but was not actively maintaining the information. This site was using data from automated passenger counters to do planning and analysis tasks.

The most integrated system found with respect to both planning and operations was King County Metro in King County, Washington. This agency does not use ARC/INFO dynamic segmentation but rather a system of related tables.

A final consideration for using ARC/INFO dynamic segmentation is the change in data structures provided by ESRI. At the time of this writing, version 8 of ARC/INFO is close to final release. Part of that release features the introduction of a true object-oriented data structure. The functionality currently offered by dynamic segmentation is likely to be replaced or significantly changed in this environment.

Based on the size and small degree of change within the Fairfax system, the team decided to conduct route building in dynamic segmentation for the pilot. Based on the success of the pilot and the change in the industry, the team will reevaluate the existing dynamic segmentation functionality before final implementation.

\section{Creating Routes}

One decision that must be made when creating routes is whether to separate routes into two paths for two-directional travel. The pilot project team found it much easier to break down a bus route into one route feature for each direction and name each by direction. PACE bus service in Chicago actually includes the direction as part of the route naming convention.

Method Applied for the Pilot Project. The initial method for creating routes was to use the Select, Makeroute, Remeasure, Subselect, and Append commands in ARC/INFO to create a bus route system. While output from this method works well, it is a tedious process. This is probably the main reason that dynamic segmentation is not used in some transit agencies.

There are two other problems associated with creating routes in this fashion. First, creating routes with loops is excessively time consuming. Figure 1 
presents an example of a loop. The route must enter and leave at point A. To create the proper measurements, the user must first build the route up to point $A$ and then use the Append and Remeasure commands for further segments of the route. This is where the project team made most of its errors in building routes. A second problem occurred when the team selected arcs to include in a particular route (see Figure 2). In the selection process it appears as though there is only one arc between points A and B. The enlargement of that area, however, shows there are really two arcs. Failing to select the smaller arc will ruin the route measurements, especially when running the Remeasure command in the loop-building process. The simple solution to avoid this is to make sure node features are turned on, but this will slow down the Redraw process when panning across the network.

Preferred Method Learned from the Process. At the end of the project, the team found that ARC/INFO's Path command provides a faster way to create transit routes than the method previously described. The Path command uses either a designated file of node locations or a user-selected set of nodes to generate route features. The Path command uses a "shortest-path" algorithm to compute a route between selected nodes. This command eliminates the problems encountered in creating routes by arc selection. Because Path uses a
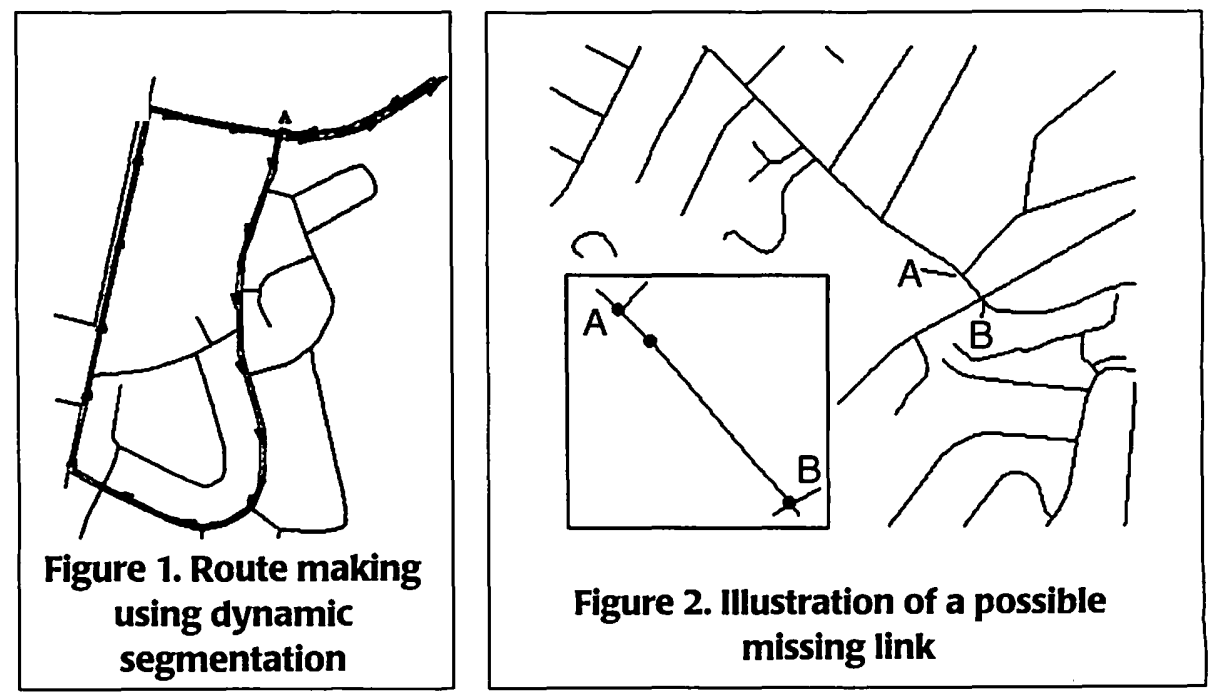

Vol. 2, No. 4, 1999 
shortest-path algorithm, there will not be any gaps in the route created. Loops are also taken care of since the stop file will be visited in sequential order. Another advantage of the Path command is that nodes used for a particular route can be saved and edited based on changes to a particular route. Recreating or altering routes becomes quite easy under this approach.

During the process, the project team also examined the possibilities of using Arc/View to create routes and stops. With the Network Analyst extension to ArcView, the user can quickly create route features using the shortestpath algorithm. Although some effort is required to link the output to the stop locations, it is an excellent and quick way to create route features. Because routes created by Network Analyst are shape files and not true ARC/INFO route systems, certain information necessary to develop comprehensive applications is missing. The project team feels the main use of ArcView and Network Analyst would be in quickly creating proposed routes for use in project analysis or for mapping purposes, while ARC/INFO would fit the best for route system maintenance.

\section{Creating Bus Stops}

Bus stop information currently exists in a paradox database. The stop location is represented by intersection attributes with "nearside" and "farside" as indications for stop distance from the intersection. The intersection attributes consisted of two fields: on-street and at-street. The results from geocoding by intersection are that the stop features are located in the middle of an intersection. Some stops are actually located 200 to 300 yards from the nearest intersection, meaning that geocoding by intersection could be misleading as to where the actual stop is located. Furthermore, during the geocoding process, a number of stops were not geocoded because, instead of containing street names, they described significant buildings (e.g., on-street: Candlewood Drive, at-street: in front of power plant).

The team evaluated the approach using a Global Positioning Systems (GPS) receiver for stop location data collection on two routes in the pilot area. With the use of a handheld GPS receiver, the team recorded accurate stop location data. The GPS receiver also allowed the team to collect field data other 
than locations. Figure 3 presents the results of the GPS data collection process. The orthophoto in the background shows the actual location of the stops collected with GPS versus the same stops geocoded by intersection. The stars in the upper-left corner of the image represent two stops geocoded by intersection attributes. The flags in the center of the image represent the actual locations of the two stops collected with GPS receivers. The flags in the lower-right corner are stops collected with GPS receivers that were not in the original stop database.

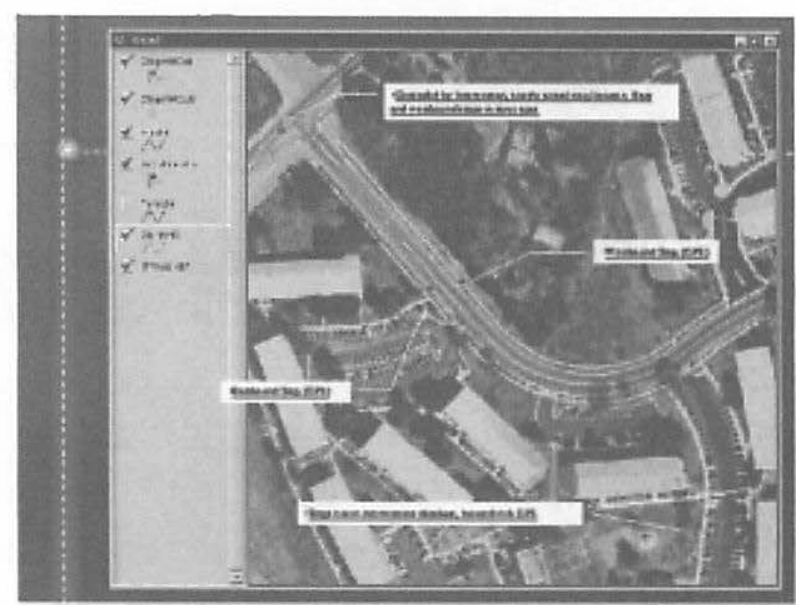

Figure 3. Comparison of stops collected by intersections and by GPS

\section{Relating Stops to Routes}

Once stop and route data are ready, they are related in ARC/INFO by the Addroutemeasure command. The only drawback to this method is that stops are related to every route within a user-specified search tolerance. For example, although five routes may pass a particular stop feature, in reality it might only be a stop for three of the five routes. It is necessary to "clean up" these extra information records. As the process is not done automatically by ARC/INFO commands, the team recommends some automation be done to carry out the task for the systemwide implementation. 


\section{Planning Applications}

Transit planning requires good knowledge of the transit services provided and the areas served. Thus, field trips have become an integral part of a transit planner's job. However, this approach in itself does not fully assist planners in acquiring an in-depth knowledge of the social, economic, land-use, and transportation features of the service area. Furthermore, field trips cannot provide a systematic ground for service justification because they are constrained by visual examination.

Desktop GIS applications can utilize and integrate data sources in the analysis and planning of transit service. Designing an automated process in ArcView could further facilitate planning analysis by supplying powerful, user-friendly tools and providing in-depth knowledge of the service area. Over the years, many transit agencies have used ArcView to develop a profile analysis of service areas. The San Diego Association of Governments developed a social-economic profile application by generating a profile of an area surrounding a transit route. They use buttons to prompt users with route number and buffer distance and develop automated spreadsheet reading of profile data.

The planning application developed by the Fairfax County DOT is unique. It creates an integrated planning process, provides planners with userfriendly tools with minimum GIS training required, and enhances the analytical function by combining decision-making processes in the design. In addition to achieving a better understanding of existing services, the application also contributes to the design of new services by assisting planners in making decisions on optimal service. The following sections describe the structure and function of the planning applications. Figure 4 illustrates the process of the planning application.

\section{Structure of Applications}

The planning application is structured into a five-step model with steps grouped under the Planning Analysis menu, as shown in Figure 5. Under the Planning Analysis menu, there are five submenus: Build New Route, Create Shed, Clip Shed, Update Info, and Export Data. Users can go through the steps in sequence depending on the type of analysis required. 


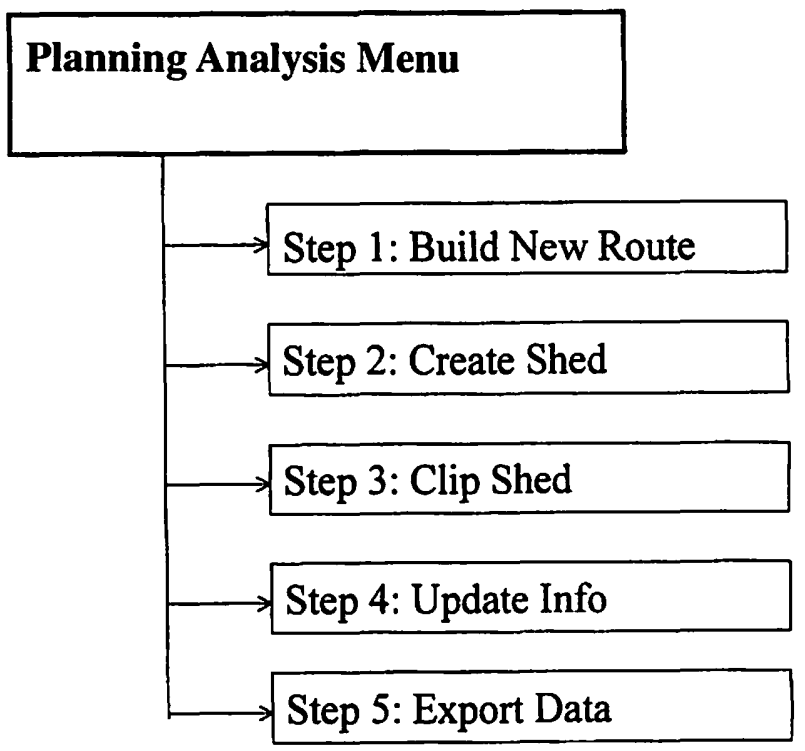

Figure 4. Planning analysis menu

\section{Analysis of Existing Service}

This analysis requires deploying only steps $2,3,4$, and 5 under the Planning Analysis menu. First, planners need to specify the shed area of the service they are studying. Starting with step 2 , they can create a shed around routes or stops within their specified buffer distance (Figure 6).

Second, planners need to find out all the transit-service-related information contained within the shed area. Using step 3, planners can clip any themes within the shed generated, including census, public facilities, and planimetric layers. However, the clipped themes contain the original attribute data in spite of the change to the original unit size. For example, in the case of census block group data, buffer areas may cover only a small portion of a block group and the clipped data actually present data for a much larger area beyond the shed. Step 4 thus functions to update data with its clipped size. By using Update Info in step 4, all relevant information will be updated based on the percentage of the original area occupied by the clipped area. The arithmetic is based on the 


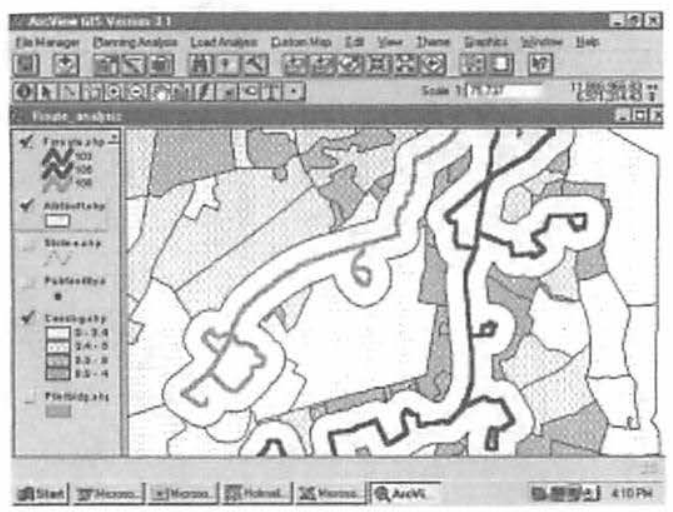

Figure 5. Menu for planning analysis

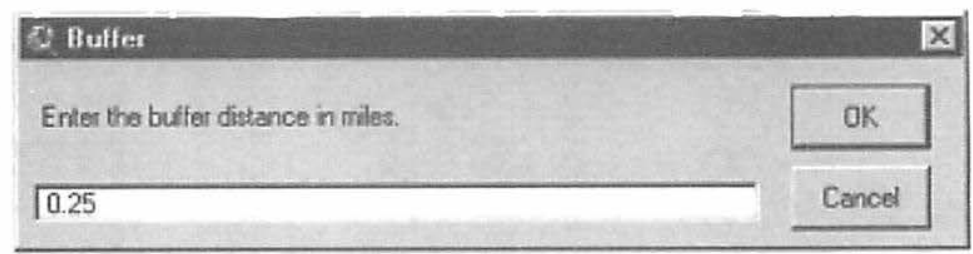

Figure 6. Dialog for creating shed

assumption that events are evenly distributed within the original spatial unit, and the Update operation should be applied only for "count" data and should not be used for density and intensity attributes. Once the data are updated, a dialog will indicate that "the table has already been updated" whenever step 4 is repeated. At this point, the user also has the option to view digital orthophotography as a means of visually verifying computed results. The unique feature of updating data with the portion of clipped area over its original significantly improves the relevance of analysis. By exporting the data in step 5, users can conduct any further (nonspatial) analysis in spreadsheet or database software.

This set of planning tools also enables planners to compare performance among routes and further analyze causes of the difference. Figure 7 presents a summary for three routes serving the Route 1 vicinity. These routes represent three different levels of performance, with Route 105 the most, Route 103 the 
moderate, and Route 108 the least productive, according to exported data from step 5 of the planning analysis. A detailed examination reveals that economic and land-use features are the underlying reasons for transit productivity along the Route 1 corridor. Route 105 serves a dense residential neighborhood with more multiple-family housing and relatively low-income level. In contrast, Route 108 is in a low-density, single-family neighborhood with the highest median household income of the three routes. Comparison of these two routes indicates that service should be enhanced in the Route 105 service area.

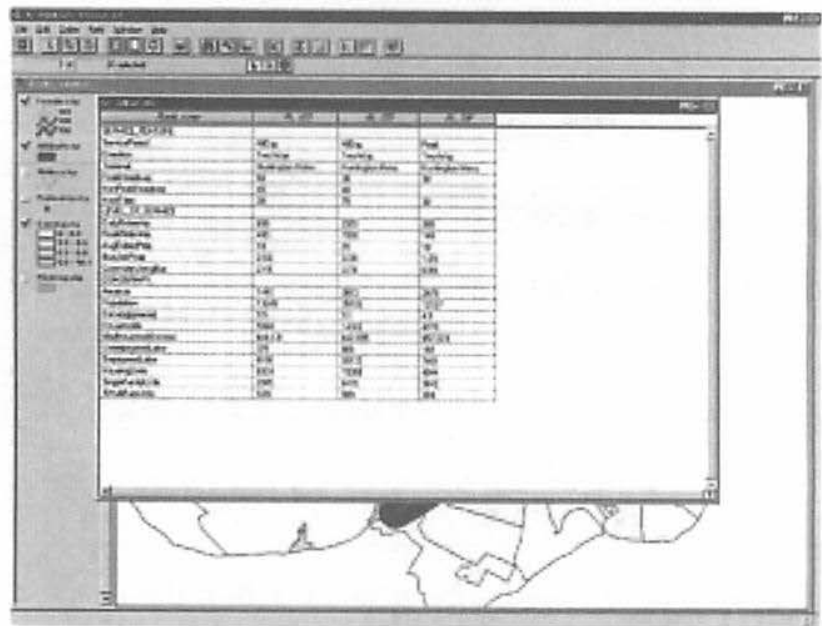

Figure 7. Summary of three routes serving the Route 1 vicinity

Furthermore, each route's information could be embedded in the application. By using hotlinks in ArcView, users can quickly find out the details of each route (Figure 8).

\section{New Route Design and Route Adjustment}

As Fairfax Connector continues to grow, new service expansion for the underserved area and adjustment of existing routes often require rigorous studies. This planning application enables planners with analytical tools to conduct scenario analysis and support decision-making in bus service design. 


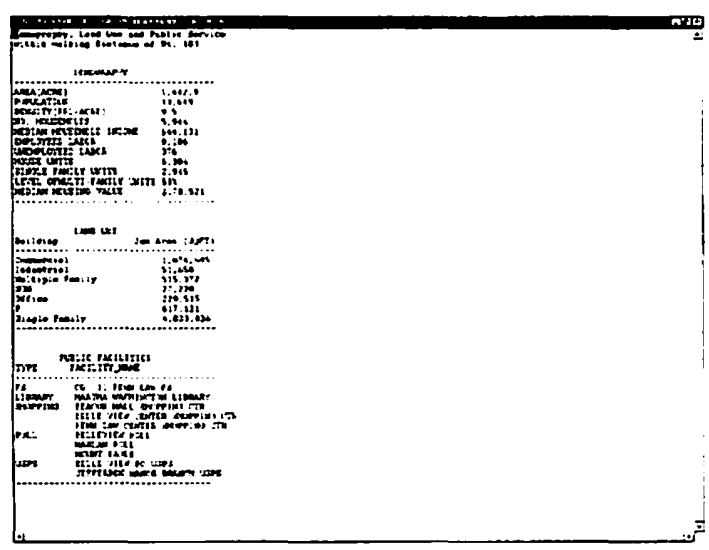

Figure 8. Summary of route information

To design the new route, planners start with step 1 under the Planning Analysis menu. The new route should follow streets on which it operates, but it does not need to be precisely overlaid above streets. After inputting the new route, planners can continue through steps 2 through 4 and quickly generate a summary of social, economic, demographic, and public service characteristics. By deploying different scenarios of new routing plans and developing service profiles for each, planners can easily compare the advantages and disadvantages of each design and select the most desirable service.

Overall, this planning application, designed in a user-friendly desktop environment, extends planners' capacity in either daily routine or major service planning. In addition to obtaining a profile of baseline features of the service, scenario analysis will play a critical role in the planning decision-making. While field observations are always essential to transit planning, the substantial amount of data presented by planning tools will further enhance the quality of transit planning. Furthermore, no additional experience is required for GIS users to carry out the five-step planning analysis, and this could encourage the acceptance of GIS in the office. Especially for medium- and large-sized transit agencies, this provides the base for successful deployment of GIS applications in day-to-day transit planning tasks. 


\section{Marketing Applications}

Several applications were identified for marketing of the Fairfax Connector system, including an Internet route query tool. As mentioned earlier, there are three other bus systems plus a portion of the Metro subway system within the study area. A true trip-planning software would ideally encompass all of these transit systems. Currently, contractors under DOT handle all passenger information inquiries and are paid by the number of calls received. A significant number of incoming calls are simple data requests such as: "What bus route passes by my house?" With a simple Internet data viewing application, the DOT could reduce the number of calls to the operations center and, in doing so, directly cut costs.

Other transit agencies have developed more comprehensive trip-planning applications, including Oregon's Tri-Met (www.trimet.org) and California's Orange County Transit Authority (OCTA) (www.octa.net). Tri-Met has several trip-planning applications. Its call-in service gives the public a complete trip plan based on an origin and destination. This trip plan is derived from the organization's GIS data. Tri-Met also provides a trip-planning component that allows the public to download route information from the Internet and perform their own trip planning. Another trip-planning application is designed for paratransit services to support citizens with disabilities. On its Internet site, OCTA provides static route maps as well as a link to the Southern California Regional Council of Governments Transtar trip planner. This software allows citizens to perform sophisticated trip planning over the Internet based on a number of user-entered parameters.

The Fairfax team developed an application using $\mathrm{MO}$ and Visual Basic to perform route information queries. MO was chosen because it could be tested in-house and converted to a web application using Map Objects Internet Map Server, Java, and HTML. The process consists of address geocoding, specifying walking distance to the bus route, and route searching. Figure 9 shows the interface for the route query application in the pilot project.

The basic premise is that users should be able to geocode their addresses and find transit information near their locations. This application takes a user- 
entered address; identifies the location with a red dot; and then displays street, bus route, and bus stop data layers. After the user clicks the Find or Find Route button, a message box pops up showing bus routes within a user-specified distance. Once a format has been decided on, the user will then have the opportunity to view route schedules and other information. The user-entered distance is a straight buffer distance. In the future, the team may add the ability to find the closest routes by network distance. Several major transit agencies, including OCTA, define stop accessibility based on network distance instead of straight Euclidean distance.

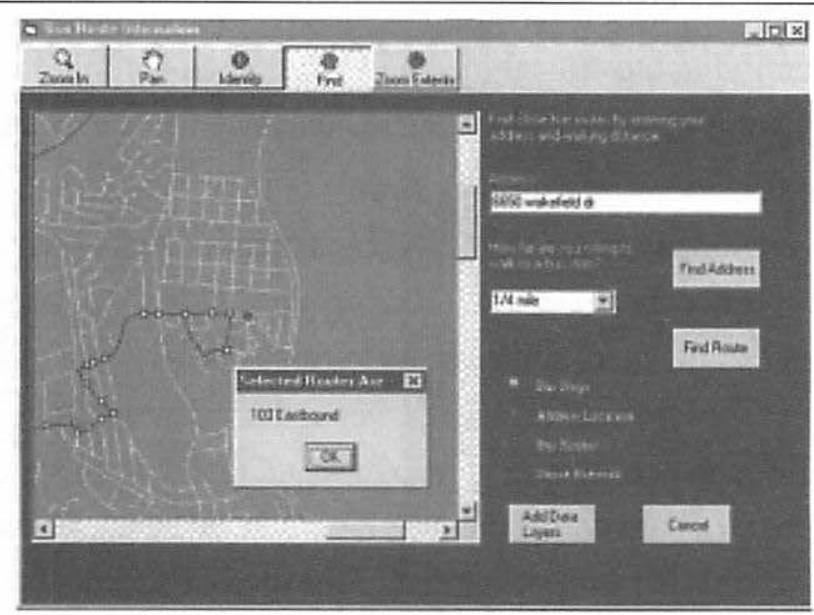

Figure 9. Illustration of route finding

Generating maps for presentations and public displays is another application identified by the marketing group. Until recently, presentation maps were done manually. Even with desktop GIS capabilities, making maps of proposed and existing routes and facilities can be time consuming. When the data conversion effort is complete, this will no longer be such a difficult task.

The marketing group also needs to display bus stops with associated attributes (e.g., shelters, benches, sign type, and whether or not a stop is a snow emergency stop). Some of this information exists and will be linked to the GPS collected stops. Information not currently available will be captured during the systemwide data collection phase. 


\section{Operation Applications}

Operation applications are designed for service monitoring and statistics. As an initial effort in application development, the project team concentrated on two areas: boarding and alighting, and route distance and running time.

\section{Analysis of Boarding and Alighting Activities}

The application provides tools for querying boarding and alighting activities by route. As shown in Figure 10, by pulling down the Operation Analysis menu and clicking on Heavy Boarding, the application will quickly query the stops and generate a table with heavy boarding activities and vice versa for the light boarding and alighting activities. This function would assist operators and planners in identifying the critical loading segments on the route and incorporate this information in service planning and enhancements.

\section{Finding Route Distance and Running Time}

Routes developed using ARC/INFO dynamic segmentation contain detailed distance information by each routing plan. This set of operation tools offers operators and planners quick access to route distance and estimated running time. Under the Operation Analysis menu, the Route Measure option will lead planners through pop-up query dialogues and report the distance measure and running time in a summary table (see Figure 11). The application for transit operations should be further explored in the area of schedule development and route statistics. More specifically, function of route statistics should be integrated later in the report to the National Transit Database.

\section{Conclusions}

The experience gained and lessons learned in the Fairfax pilot system reported here provide valuable insights in designing systemwide implementation and are instrumental in deciding the future direction of the project.

\section{Baseline Database Development}

The base required for the Fairfax project includes route and stop database and coverage. Although the team started with manual dynamic segmentation after trying several other approaches, at the end they found the Path command in ARC/INFO a most effective approach in building bus routes. It will ulti- 


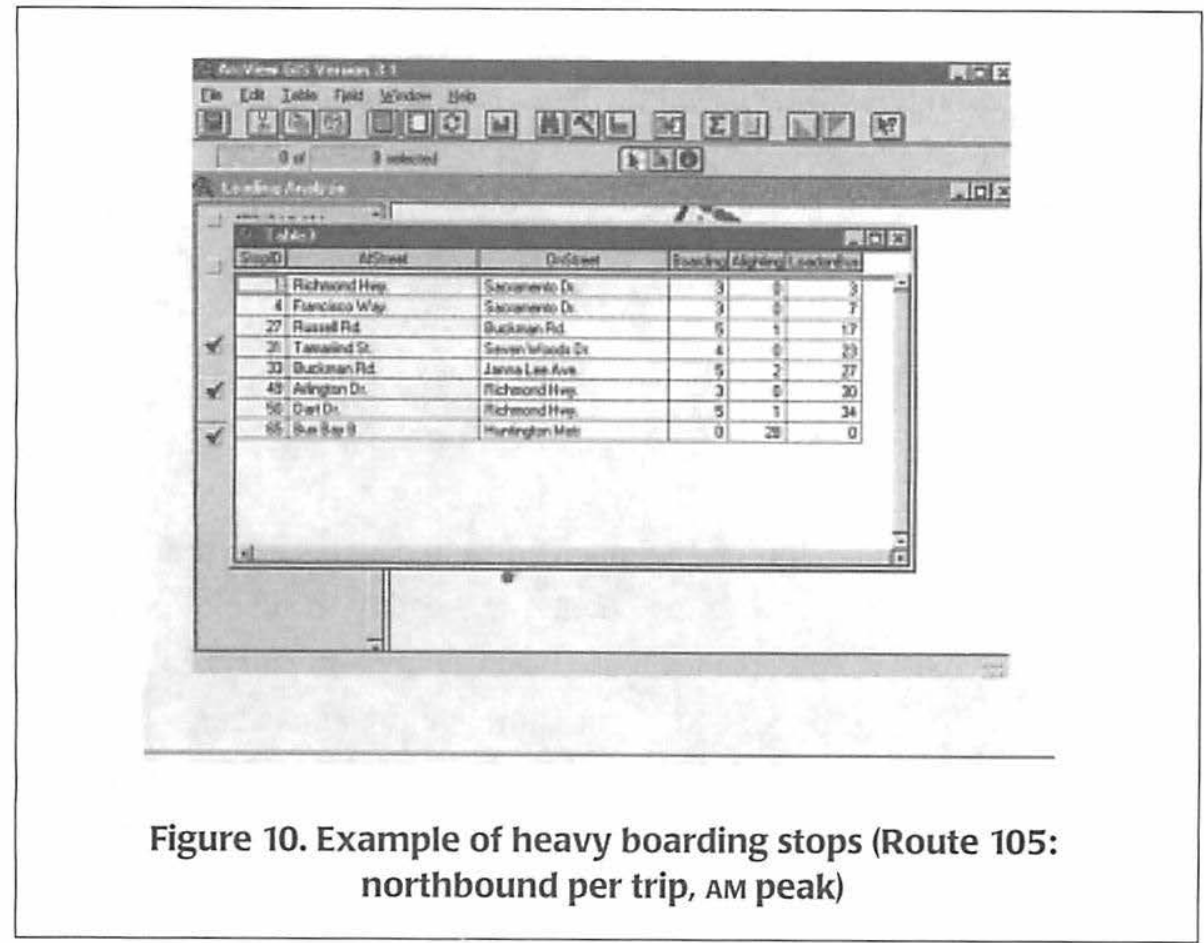

mately facilitate the maintenance and updating of bus route systems by DOT staff with some training and assistance from the GIS staff.

The existing stop data using intersection as a location indication does not reach the required accuracy. GPS equipment should overhaul the current stops

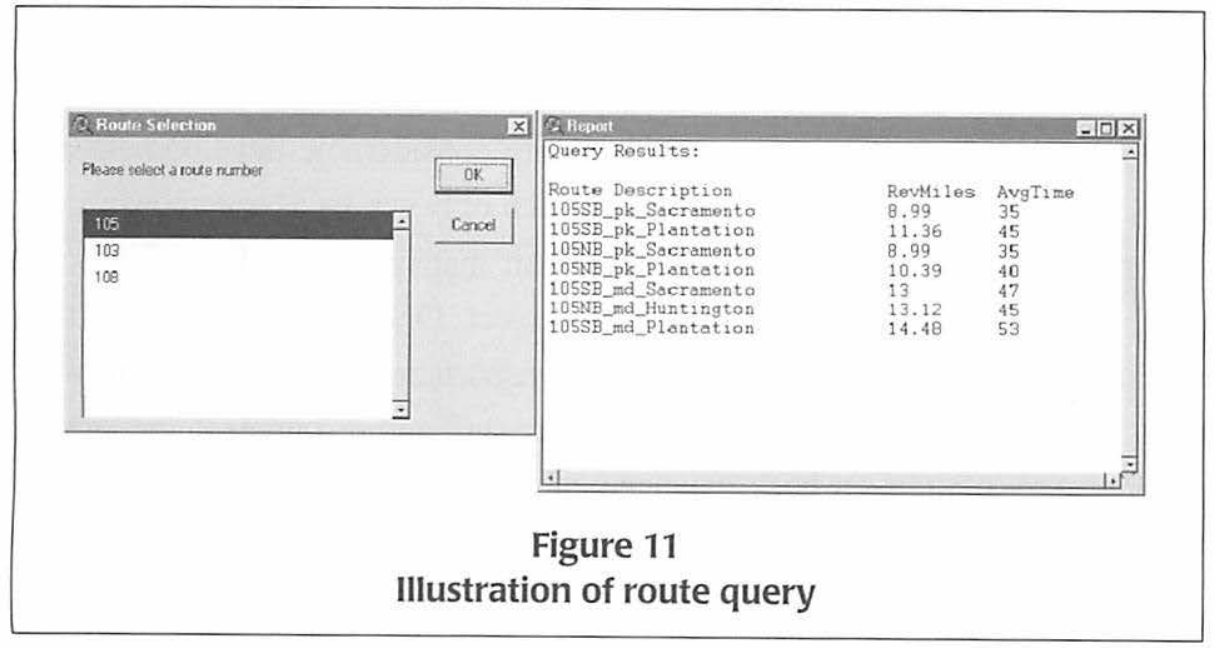


database in the systemwide implementation of the project. Existing information will be linked to GPS collected points. There is an ongoing effort by the Transit Standards Consortium to compile a set of recommended practices for bus stop data collection (www.tsconsortium.org), which could be used as a guide for future stop database collection. Transportation professionals have participated in teleconferences to identify methodologies, attribute items for collection, known problems, and many other issues associated with bus stop data collection.

\section{Data Consistency}

The Fairfax County DIT has put much effort in data collection and development. The data compiled by the County are readily available to various agencies. In the Fairfax case, the team used the County's street centerline coverage, planimetric data, orthophoto layer, and public facility data. However, they were not able to use the County's census data because the County developed it as a subcensus tract, which is different from a standard census tract. As a result, the various census data could not be used on the County-defined subcensus tracts. A decision was made at the beginning to use census block-group data, which were downloaded from the Census Bureau. Also, for the transit data, the team used data from different collection sources and no continuity could be guaranteed for the future. As the project goes into system implementation, future maintenance and update require consistency and continuity of all the data applied.

\section{Funding and Resources}

A system implementation would require considerable development and refinement of the existing pilot project, which only serves as a demonstration in terms of the role of GIS in routine transit management. Thus, resources, staff, and time would be needed for the project. One large constraint is that funding is not readily available to local transportation agencies dealing with daily system management. The need for more staff and resources in the face of funding issues could set back management incentives for systemwide implementation. 


\section{Training and Software}

Fairfax County DIT periodically offers basic GIS training to County employees. As the applications designed target minimum user experience, the training provided should be sufficient for each section in the transit operations division to leverage the capabilities of GIS technology. However, one current problem is that the DOT only has a couple of licenses for ArcView. One possible solution considered by the GIS department is to offer ArcView via a CITRIX Metaframe application. This would allow users at remote locations to access ArcView on a central server. The County currently has 80 licenses of ArcView deployed throughout the region. Serving these licenses through Metaframe would allow the County to reach more end-users. With this option the GIS department would be able to better serve transportation professionals interested in applying the planning, operations, and marketing applications.

Overall, the applications designed for day-to-day planning, marketing, and operations in the Fairfax DOT set a prototype of GIS in transit management with its unique desktop features. While the applications demonstrate potentials of GIS in transit, they will be further refined for the final system implementation based on staff comments. Once all the routes are developed, it would be just a plug-in to link the already designed application interface with the route and stop data.

\section{About the Authors}

WENYU JIA, (wjiaØl@co.fairfax.va.us) a transportation planner with the Fairfax County Department of Transportation, specializes in transit planning and operations. She holds a Master of Science in transportation engineering and a Master's degree in city planning from the University of California at Berkeley. Ms. Jia has been with the Fairfax County Transportation Department for almost two years.

BRENDAN FORD (Brendan.Ford@co.fairfax.va.us) is the team leader for the Application Development section of the Fairfax County Department of Geographic Information Services. He graduated in 1987 from Virginia Polytechnic Institute and State University with a bachelor's degree in geography and in history. He has been employed by Fairfax County for 11 years. 


\title{
A Transit Access Analysis of TANF Recipients in Portland, Oregon
}

Thomas W. Sanchez, Portland State University

\begin{abstract}
Little evidence exists regarding the relationship between transit service availability and the ability of welfare recipients to find stable employment. While policymakers continue to assert that increased public transit mobility can positively affect employment status, there is little empirical evidence to support this theory. It is generally assumed that public transit can effectively link unemployed, carless persons with appropriate job locations. From these assumptions stems the common belief that if adequate transit were available, the likelihood of being employed would increase. Hence, the call for more transit services to assist moving welfare recipients to gainful employment. Current available evidence is anecdotal, while general patterns of transit access and labor participation remain relatively unexplored.

This analysis examines whether transit access service is less available to Temporary Assistance for Needy Families (TANF) recipients in the City of Portland, Oregon. It uses disaggregate TANF recipient location data from the State of Oregon Department of Adult and Family Services (AFS); transit route/stop data from Tri-Met; block-group census data; and disaggregate employment location data within Geographic Information Systems (GIS). GIS capabilities are essential in performing
\end{abstract}


network accessibility analyses and for analyzing spatial patterns of TANF recipient and employment locations. The results of this analysis provide an assessment of the availability and quality of transit service for TANF recipients.

\section{Introduction}

The Personal Responsibility and Work Opportunity Reconciliation Act of 1996 is a renewed effort to move persons from welfare rolls to stable employment. This legislation attempts to provide states with more flexibility in assisting low-income households and also to provide incentives for states to reduce welfare caseloads. The revised system is administered through the TANF program (formerly Aid to Families with Dependent Children), which institutes increasingly severe time restrictions and qualifying criteria (Danziger et al. 1999).

Recognizing the fact that most of the households within the TANF program have limited transportation mobility, $\$ 750$ million was allocated over five years for job access and reverse commute programs (Surface Transportation Policy Project 1998). Because low-income households have especially low rates of automobile ownership, with many having no access to an automobile, they depend on public transit, which then affects the locations and types of employment that are available to them (Murakami and Young 1997; Coulton, Leete, and Bania 1997). Some argue that public transit is not a viable alternative to the personal automobile due to the extent of geographic imbalance between housing and jobs (Wachs and Taylor 1998; Ong and Blumenberg 1998). The result is a significant challenge routinely faced by transit operators: to provide effective service despite increasing automobile dependency as well as dispersed and transit-inaccessible land-use patterns. The spatial mismatch between the residential location of low-income, urban households and the location of new low-skill jobs has received considerable attention in the academic literature (Ihlanfeldt and Sjoquist 1998).

Transit agencies have faced reduced ridership and revenue amid simultaneous demands for new service to dispersed employment in the suburbs that is inherently more costly to provide. Transit service also operates in a political 
environment where the costs of marginal increases in service levels may encounter opposition due to competing objectives from other public agencies (Wachs 1995). If urban service is less utilized than it once was, but is still desired by remaining transit customers, it is difficult to sustain political arguments to provide new services where the current demand is smaller or latent and the automobile is the focus of land-use development activities.

While transit routes are designed, in part, to serve worktrips in urban areas, little evidence exists regarding the relationship between transit service provision and labor participation rates. Policy-makers continue to assert that employment status is a function of transportation mobility, despite limited empirical evidence to support this theory. It is generally assumed that public transit can effectively link unemployed, carless persons with appropriate job locations. The common belief, based on these assumptions, is that adequate public transit increases a worker's likelihood of being or staying employed (U.S. Department of Transportation 1998). Hence the call for more transit services to assist moving welfare recipients to stable employment. Available evidence to date is anecdotal; general patterns of transit access and labor participation are now becoming the focus of many analyses-especially with the use of GIS.

GIS is being used increasingly to better understand the spatial dimension of where TANF recipients live and the location of appropriate job opportunities. A range of research has operationalized employment and transit accessibility measures for low-wage workers (Community Transportation Association of America 1998; Lacombe and Lyons 1998). In most cases, the use of GIS is limited to mapping concentrations of TANF recipients and job opportunities rather than being used for spatial analyses to generate solutions to associated transportation planning problems. Some have utilized more advanced methodologies that empirically examine the spatial relationship between jobs, transit, and employment outcomes (Shen 1998; Thompson 1997; Sanchez 1999). The use of GIS will likely increase for these purposes as there is further recognition of the benefits of geographical analysis. 


\section{Hypotheses}

This analysis examines three hypotheses for TANF recipients in the City of Portland, Oregon. These hypotheses address whether TANF recipients experience lower levels of transit and employment access compared to the overall population of workers. The three hypotheses are:

1. TANF recipients have less physical access to transit stops compared to other transit commuters.

2. TANF recipients live in areas with less frequent transit service compared to other transit commuters.

3. TANF recipients have less transit access to entry-level employment locations compared to other transit commuters.

The first hypothesis concerns the level of transit access available to TANF recipients. Transit access is typically considered adequate if persons live within a 0.25 -mile walking distance to the nearest transit stop (Urban Mass Transportation Administration 1979). The implicit assumption is that their final destination is also within walking distance to a transit stop. Beyond 0.25 mile, the time cost and inconvenience usually inhibit transit usage. The mean walking distance to the nearest transit stop for TANF recipients is compared to the mean distance from block-group centroids. The centroid represents the "average" location of residents within each block group. For comparison purposes, each centroid is weighted by the number of workers reporting that they use transit to get to work (from the 1990 census). If the average walking distance to the nearest transit stop for TANF recipients is greater than that of other transit commuters, it would indicate that transit is less accessible to TANF recipients.

The second hypothesis considers the quality of transit service. Along with physical proximity to stops, service frequency also has a significant affect on ridership (Black 1995). Mobility levels increase when riders are not limited by infrequent or unreliable transit availability. To test the second hypothesis, the mean peak scheduled service frequency (7 A.M. to 9 A.M..) at the nearest transit stop for all TANF recipients is compared to the mean service frequency for frequent transit commuters (by block group). If the service frequency for TANF recipients is significantly less than that of transit users, the utility of 
transit for recipients will be lower than for locations with more frequent service. The measures of service quality and proximity provide useful indicators of whether TANF recipients are at a disadvantage in terms of mobility given that their vehicle ownership rates are very low.

The third hypothesis focuses on issues related to the spatial mismatch hypothesis and job accessibility that are central to welfare-to-work initiatives. One solution to the spatial mismatch of worker and job locations is to increase transportation mobility levels, especially relative to entry-level employment locations. The underlying assumption is that shifts in new employment locations have produced a geographic separation between residences and jobs that has contributed to higher levels of employment instability. For this reason, if job accessibility increases through improved public transit services, then labor participation levels should increase. The measures of job accessibility for TANF recipients include only entry-level positions because recipients have generally low levels of educational attainment and job skill levels (Immergluck 1998). The mean number of retail and service employment locations (considered to be entry level or low skill) that can be reached using transit is used to test whether TANF recipients have lower levels of transit access to these jobs compared to other employed persons that rely on transit.

\section{Methodology}

The AFS provided an address database for TANF recipients in the Portland metropolitan area. A total of 5,186 out of 5,286 records were geocoded for the City of Portland. Of the 100 unmatched records, 92 could not be matched to street addresses because the recipient was homeless or listed a post office box for their home address. Along with street addresses, AFS provided demographic characteristics about each recipient and their current status. In summary, approximately half $(56.6 \%)$ of Portland TANF recipients are white and predominantly female (79.6\%). On average, recipients are 32 years old with less than 11 years of education. More than half of TANF recipients have received assistance for 12 months or less while approximately 20 percent have received assistance for more than four years.

Along with the disaggregate TANF recipient location data, the analysis 
uses transit route and stop data from Tri-Met; 1990 block-group census data; and disaggregate employment location data within a GIS. GIS capabilities are essential in performing network accessibility analyses and for analyzing spatial patterns of TANF recipient and employment locations. For each TANF recipient location and block-group centroid the distance along the street network to the nearest transit stop is used as an estimate of walking distance. This assumes that pedestrian facilities are available along each street segment. Similarly, the average peak-hour service frequency at the nearest stop (in terms of minimum walking distance) is assigned to each TANF and block-group centroid location. Both the walking distance and service frequency measures assume that persons use the nearest transit stop location-which may be true in most, but not all cases.

For service and retail job locations, an employment accessibility index was calculated for each TANF recipient and block group. Recipient locations and block-group centroids were used as trip origins with the locations of service and retail jobs as destinations. An average total travel time of 60 minutes with 10-minute penalties for transfers was used for job accessibility calculations. Significantly lower levels of job access for TANF recipients may suggest that spatial mismatches are a factor contributing to low rates of labor participation. The accessibility calculations are based on the following equation and estimated using a GIS:

where:

$$
P_{i}=\sum_{j=1}^{n} W_{j} d_{i f}^{-\beta}
$$

$P_{i}$ is the employment accessibility of TANF recipient or block group $i$.

$W_{j}$ is the number of jobs within walking distance of each transit stop j.

$d_{i j}$ is the travel time between $\mathrm{i}$ and $\mathrm{j}$.

$\beta$ is the exponent for distance decay ( 2 used for this analysis).

$n$ is the number of transit stops in the study area.

\section{Results}

Statistical tests were used to determine if a significant difference exists 
between mean values of transit access and employment access for TANF recipients compared to other transit commuters. Mean tests were also applied to the employed population for comparison purposes. The tests were conducted by comparing the mean values for TANF recipients to the mean values for block groups (weighted by the number of workers using transit for worktrips and also weighted by the total number of employed persons for each block group). The statistical results suggest that on average, TANF recipients live slightly closer to transit services than do other frequent transit commuters (Table 1). The average distance to the nearest stop for TANF recipients is also less than that of the overall employed population. This indicates that TANF recipients do not suffer disproportionately from poor physical access to transit routes.

\begin{tabular}{|lcccc|}
\hline \multicolumn{5}{|c|}{ Table 1 } \\
& \multicolumn{5}{c|}{$\begin{array}{l}\text { Standard } \\
\text { Deviation } \\
\end{array}$} & \multicolumn{5}{c}{ Mean } & $(S D)$ & $N$ & t-test $^{\mathrm{a}}$ \\
\cline { 2 - 5 } & & & & \\
TANF & 0.185 & 0.124 & 5,185 & - \\
Transit commuters & 0.196 & 0.128 & 20,616 & $<.005$ \\
All workers & 0.249 & 0.203 & 236,634 & $<.005$ \\
\hline
\end{tabular}

a. Two-tail significance, $t$-test for equality of means (compared to TANF observations).

While TANF recipients in Portland appear to live in areas with nearby transit services, the frequency of scheduled service near them tends to be slightly less than that of frequent transit commuters (Table 2). The average difference in service frequency is approximately 0.5 minutes, while the overall employed population averages approximately 2 minutes less frequent service than do TANF recipients. While transit service frequency is an important indicator of service quality, the 0.5 -minute average difference with transit commuters does not represent a distinct disadvantage for TANF recipients.

Walking distance to the nearest transit stop and service frequency at the nearest stop serve as transit system access measures. Transit access will only 
Table 2

Peak Service Frequency at Nearest Transit Stops (mins)

\begin{tabular}{llll}
\hline Mean & $S D$ & $N$ & $t$-test \\
\hline
\end{tabular}

$\begin{array}{lrrrc}\text { TANF } & 17.312 & 7.431 & 5,185 & - \\ \text { Transit commuters } & 16.827 & 7.866 & 20,616 & <.005 \\ \text { All workers } & 19.355 & 10.795 & 236,634 & <.005\end{array}$

be beneficial if the route network increases overall accessibility to employment opportunities. In this case, the measures of relative access to entry-level job locations are an indicator of route system effectiveness. The comparison of mean employment access through the transit network indicates that there is no significant difference between TANF recipients and frequent transit commuters (Table 3). In addition, the results indicate that there is no statistical difference in employment access between TANF recipients and the overall employed population. In general, TANF recipients do not appear to be at a particular disadvantage in terms of reaching employment locations using transit compared to other transit commuters.

Figure 1 shows the geographic distribution of TANF recipients in the City of Portland. Concentrations of recipients are apparent in the north and northeast portions of the City. The map also shows the correlation between recipi-

\begin{tabular}{|c|c|c|c|c|}
\hline \multicolumn{5}{|c|}{$\begin{array}{l}\text { Table } 3 \\
\text { Relative Accessibility to Entry-Level Jobs }{ }^{a}\end{array}$} \\
\hline & Mean & $S D$ & $N$ & $t$-test \\
\hline TANF & $2,318.3$ & $17,457.4$ & 5,104 & - \\
\hline Transit commuters & $2,412.1$ & $17,173.0$ & 20,422 & .728 \\
\hline All workers & $1,956.1$ & $16,689.7$ & 232,497 & .126 \\
\hline
\end{tabular}

a. Calculated as the combined accessibility to service and retail employment locations. 
ent locations and census block groups ranked by the transit and employment accessibility variables discussed previously. The block group rank for walking distance to the nearest bus stop (a high rank represents closer locations), service frequency at the nearest stop (a high rank represents higher frequency), and employment accessibility (a high rank represents higher accessibility) are added together for a composite rank. The highest values (dark shade) shown on the map represent the areas with the worst relative transit and employment access ( 2 standard deviations above the mean). The correlation between a block group's rank and the presence of TANF recipients (percent of the blockgroup population that are recipients) is not significantly correlated $(R=.0069$, $p=.441$ ). In fact, the area with the highest concentration of TANF recipients also has high levels of transit and employment accessibility. Such findings are relatively common, either from the standpoint of service delivery bias or through spatial constraint (McLafferty 1982).

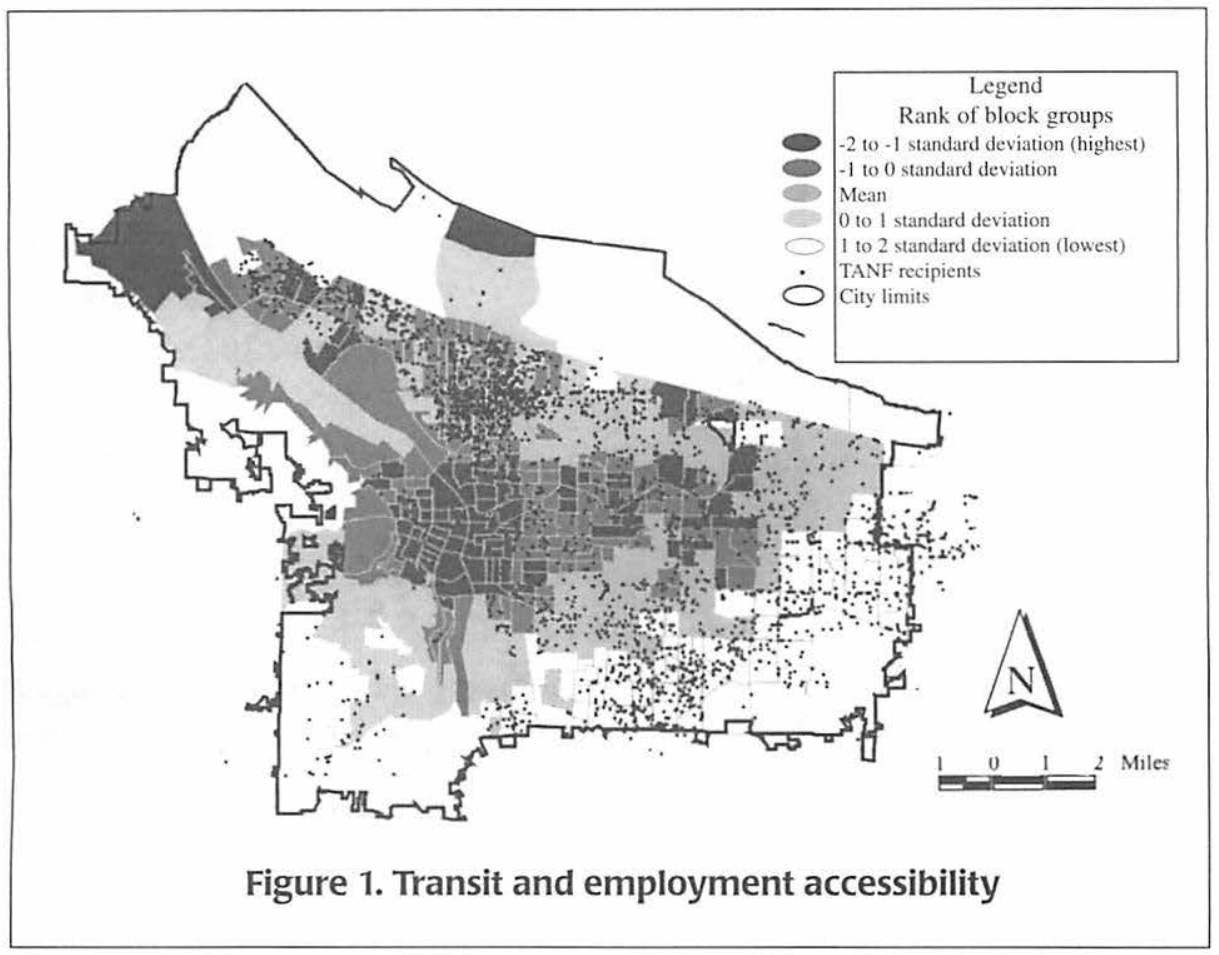




\section{Conclusions}

The three hypotheses examined in this article focus on the relative availability of transit services throughout the City of Portland. Because 85 percent to 90 percent of TANF recipients do not have access to an automobile for personal use, their mobility needs have to be met by alternative means (U.S. DOT 1998). The results of this analysis indicate that TANF recipients realize levels of transit and employment accessibility similar to those of employed transit commuters. An explanation for this is that mobility needs are complex and may not be satisfied by conventional increases in public transit service such as additional routes or increased service frequency.

In order to meet the mobility needs of low-income and unemployed persons, a variety of strategies are being implemented. For example, the Joblinks demonstration program in 10 U.S. cities used a variety of transportation services including demand-responsive van service, fixed-route reverse commute express bus service, school buses, volunteer carpools, demand-responsive taxi, and extended-hour demand-responsive transit (Goldenberg, Zhang, and Dickson 1998). Effective mobility strategies will need to provide high levels of service beyond the traditional A.M. and P.M. peak hours, for late-night to early-morning shifts. With conventional transit service, workers relying on transit may be able to reach their work locations by transit but service may not be available when their shifts are over. This is where demand-responsive and extended-hour services can be especially valuable in meeting specific employment-related mobility needs.

Public transit can better respond to welfare-to-work challenges if it can provide "collaboration among transportation, employment, and other human services organizations" (U.S. General Accounting Office 1998). Public transit planners are recognizing that employers must be involved in the design and implementation of work-related transportation strategies. Human service agencies also understand the transportation mobility needs of TANF recipients that extend beyond employment-related travel. To effectively address these transportation mobility needs, other destinations that are part of daily travel needs (e.g., shopping, school, childcare, healthcare, and job-training locations) must 
also be considered. Because a vast majority of TANF recipients do not own cars, simply providing transportation to work and back only meets a portion of their daily travel requirements.

Further research is needed that combines measures of employment accessibility with other measures of access to shopping, schools, and daycare center locations to better assess overall transportation mobility needs. In addition, similar analyses need to be performed in a variety of urban locations so that generalizable results can be obtained. Mobility strategies may need to place more emphasis on these nonwork locations to meet the daily travel needs of the low-income population and persons seeking employment. Alternatively, further research may indicate that publicly provided transit services do not significantly affect TANF recipient employment opportunities. In this case, resources should be coordinated to address other contributing factors including education, job training, childcare, health services, and affordable housing.

\section{References}

Black, A. 1995. Urban mass transportation planning. New York: McGraw-Hill, Inc. Community Transportation Association of America. 1998. Access to jobs: A guide to innovative practices in welfare-to-work transportation. Washington, DC: CTAA.

Coulton, C., L. Leete, and N. Bania. 1997. "Housing, transportation and access to suburban jobs by welfare recipients in the Cleveland area." Center for Urban Poverty and Social Change, Mandel School of Applied Social Sciences, Case Western Reserve University.

Danziger, S., M. Corcoran, S. Danziger, C. Heflin, A. Kalil, J. Levine, D. Rosen, K. Seefeldt, K. Siefert, and R. Tolman. 1999. Barriers to the employment of welfare recipients. Poverty Research and Training Center, University of Michigan.

Goldenberg, L., J. Zhang, and C. Dickson. 1998. “Assessment of the Joblinks demonstration projects: Connecting people to the workplace and implications for welfare reform." Paper presented at the 1998 annual Transportation Research Board Meeting, Washington, DC.

Ihlanfeldt, K. R., and D. L. Sjoquist. 1998. The spatial mismatch hypothesis: A review of recent studies and their implications for welfare reform. Housing Policy Debate 9(4): 849-892. 
Immergluck, D. 1998. Job proximity and the urban employment problem: Do suitable nearby jobs improve neighborhood employment rates? Urban Studies 35: 7-23.

Lacombe, A., and W. Lyons. Spring 1998. The transportation system's role in moving welfare recipients to jobs. Volpe Transportation Journal (2): 1-9.

McLafferty, S. 1982. Urban structure and geographical access to public services. Annals of the Association of American Geographers 72(3): 347-354.

Murakami, E., and J. Young. 1997. "Daily travel by persons with low income." Paper presented at the National Personal Transportation Survey Symposium, Bethesda, Maryland.

Ong, P., and E. Blumenberg. 1998. Job access, commute and travel burden among welfare recipients. Urban Studies 35(1): 77-93.

Sanchez, T. W. 1999. The connection between public transit and employment: The cases of Portland and Atlanta. Journal of the American Planning Association 65(3): 284-296.

Shen, Q. 1998. Location characteristics of inner-city neighborhoods and employment accessibility of low-wage workers. Environment and Planning B 25: 345-365.

Surface Transportation Policy Project. 1998. Getting to work: The access to jobs and reverse commute programs. Progress VIII(4): 10.

Thompson, G. L. 1997. How ethnic/racial groups value transit accessibility: Modeling inferences from Dade County. Paper presented at the annual meeting of the American Collegiate Schools of Planning, Ft. Lauderdale, Florida.

Urban Mass Transportation Administration. 1979. Analyzing transit options for small urban communities-Analysis methods. Washington, DC: U.S. Department of Transportation.

U.S. Department of Transportation. 1998. Bureau of Transportation Statistics. Welfare reform and access to jobs in Boston. Report BTS -98-A-02. Washington, DC.

U.S. General Accounting Office. 1998. "Welfare reform: Transportation's role in moving from welfare to work." GAO/RCED-98-161, Washington, DC.

Wachs, M. 1995. "The political context of transportation policy," in S. Hanson, (ed.), The geography of urban transportation. New York: The Guilford Press, 269-286. Wachs, M., and B. D. Taylor. 1998. Can transportation strategies help meet the welfare challenge? Journal of the American Planning Association 64(1): 15-19. 


\section{About the Author}

Thomas W. SANChez (sanchez@pdx.edu) is an assistant professor of urban studies and planning and a research associate in the Center for Urban Studies at Portland State University. His research focuses on the social and spatial impacts of transportation planning. 


\title{
Evaluating Transit Market Potential and Selecting Locations of Transit Service Facilities Using GIS
}

\author{
Srinivas S. Pulugurtha and Shashi S. Nambisan, \\ UNLV Transportation Research Center \\ Nanda Srinivasan, Cambridge Systematics Inc.
}

\section{$\overline{\text { Abstract }}$}

Accessibility to transit service facility (TSF) locations plays a significant role in the success of public transportation systems. The ease with which the end-user can reach a TSF (e.g., bus stops, rail stations, or multimodal centers) plays prominently in the decision-making process of the individual.

This article presents a working definition for transit market potential based on accessibility in terms of walking distance and walking time. Further, a measure is constructed to evaluate transit market potential for TSF locations for a transit system. The measure of transit potential is represented by an index value based on demographic criteria such as employment, household size, vehicle ownership, etc. This index can be used to identify locations of TSFs that increase a route's potential for ridership. A methodology is proposed to estimate the Index of Transit Potential for TSFs. This methodology involves (I) identifying the accessible network of streets around each TSF that is within an acceptable access threshold for a transit rider, and (2) estimating the transit market potential based on key demographic characteristics. The analytical and visualization capabilities of a Geographic Information Systems (GIS) 
program are utilized to help attain the objective. A case study is used to demonstrate the application of the methodology. In the case study, a portion of a route of the Las Vegas Citizens Area Transit (CAT) system is analyzed and the Index of Transit Potential is estimated. The index values are then used to locate TSFs along the route. This is compared with the existing stop locations for the route.

\section{Introduction}

Increasing congestion on roadways is a problem of concern not only to transportation system managers but also to elected officials, public administrators, and the general public. Potential demand management solutions to alleviate congestion include encouraging carpooling, promoting transit usage by providing effective public transportation systems, and reducing vehicle trips in general. The success of any public transit system depends on several factors including service frequency, fares, reliability, TSF locations, accessibility, comfort, convenience, and safety. The spacing and location of TSFs are major determinants of system availability and reliability (Regional Transportation Commission of Clark County [RTC] 1997). To enhance passenger convenience and to ensure desirable operating speeds of buses, transit agencies develop guidelines for spacing stops on a route. For example, the stop spacing identified in guidelines used by the CAT system operating in the Las Vegas, Nevada, metropolitan area ranges from 152 to 213 meters (500 to $700 \mathrm{feet}$ ) in high-density residential locations and 335 to 457 meters $(1,100$ to 1,500 feet) in low-density residential locations. However, such guidelines are based on general rules of thumb. This article presents a methodology to support developing such guidelines and to locate TSFs.

\section{Background}

The primary criteria that influence the locations of TSF include accessibility to potential passengers, safety, transit route configuration (grid or radial network, express or local route, etc.), and traffic operations. Consistency in TSF locations, especially for transit systems that share the right-of-way with other vehicles, minimizes confusion for potential patrons, transit operators, and for other vehicles. Typically, nearside, farside, and midblock locations are possible 
for stops on transit facilities. In the Las Vegas metropolitan area, bus stops are normally located at the farside of an intersection approach since most of the stops are on through travel lanes (RTC 1997). This decision was made in order to minimize delays to other vehicles at the intersection because of signal-timing considerations. Other advantages of farside stop locations for a bus transit system include the following (RTC 1997):

- Passengers boarding and alighting are less likely to cross in front of the bus.

- Reduced interference with traffic at intersections where there are heavier traffic volumes on the approach than on the departure leg.

- Stopped buses do not obstruct sight lines to the left for vehicles entering the intersection from a side street.

- Sight distance is improved for pedestrians.

Another general criterion includes avoidance of proximity to driveway or alleyways.

Typical transit systems have three main types of TSFs: transfer stops, time-check stops, and other general stops. In practice, sites of transfer stops are decided based on the network configuration. Time-check stops are TSFs where transit vehicles stop regardless of whether there are passengers to board or alight. The intent of time-check stops is generally to facilitate schedule adherence (to the extent possible) along sections between such stops. The general stops are TSFs at which the transit vehicles stop only if there are boarding or alighting passengers. This article addresses the location of the general stops or general TSFs.

In order to facilitate time-coordinated transfers for passengers between various routes, it would be advantageous to establish a transfer TSF also as a time-check TSF. Then, if the schedules of routes that traverse this site are coordinated, it would minimize the delays to passengers transferring between these routes. There could be a minimum of 4 transit routes operating at a site that is the intersection of 2 two-way streets (or rail lines). The spatial and temporal distributions of the transit passenger flow would influence the decision to coordinate none, some, or all of the routes at this site. The decision to locate a TSF 
at a transfer point should consider the walking distance, walking time, and the transit schedule (scheduled departure time). Such transfer and time-check TSFs typically account for a very small portion of all the designated stops along a bus route. For example, there are 6 intermediate time-check stops that also are transfer stops along Route 101 of the CAT system. This route has a total of 28 stops in the southbound direction (i.e., transfer and time-check stops account only for about 21 percent of all the stops). Thus, on a typical transit system, the majority of the TSFs are those termed "general stops." They need to be located based on factors and considerations that are in addition to schedule coordination issues.

\section{Review of the Literature}

A recent analysis based on the 1995 Nationwide Personal Transportation Survey (NPTS) showed that transit's share in urban areas larger than 3 million persons is 3.77 percent, while for urban areas with 0.5 million to 1 million persons it averages 0.88 percent (Chu 1998). However, transit's share of trips by persons with annual household incomes less than $\$ 15,000$ or living within one block of a bus stop are 11.75 percent and 7.96 percent, respectively, for areas larger than 3 million persons, and 2.19 percent and 2.26 percent, respectively, for areas with 0.5 million to 1 million persons. These statistics reflect the need to approximately locate TSFs if the objective is to increase the ridership. The focus of this article is to identify the best (or good) locations of TSFs based on accessibility and transit market potential.

Accessibility, or access opportunity, is defined as "the spatial quality of the relation between location of infrastructure facilities and the location of the users" (Bach 1981). Thus, it is the ease with which TSFs (say, bus stops) can be reached by a given population set. Distance and time are two measures of accessibility. A facility is considered accessible to the user if the accessibility measure is less than an acceptable, predefined maximum value.

Henk and Hubbard (1996) state that transit service coverage refers to the spatial proximity of transit service throughout an urban area. They evaluated potential by including a variety of transit system characteristics measured by 
urban area demographic characteristics such as population, urbanized land area, and population density. Bach (1981) concluded that the type of distance measure and the level of aggregation influence the indices of accessibility and access opportunity.

GIS has traditionally been used in analysis, postprocessing of results, and visual representation of data to facilitate easier recognition of spatial correlation between data and allow for easier decision-making (Environmental Systems Research Institute, Inc. 1997). O’Neill et al. (1992) described a procedure for performing service area analysis on transit routes using a GIS software program. A route's accessibility was indicated by the total number of persons living within the service area. Gomez and Zhao (1998) presented a GISbased methodology that improves the estimate of pedestrian access to transit by utilizing street network information, land-use data, and household information from census data.

Johnston (1966) studied important aspects of transit service and settlement patterns by constructing an index of accessibility. Henk and Hubbard (1996) state that the index of accessibility should be a measure based on various transit system characteristics. Evaluation of accessibility indices for transportation networks have been presented by Sathisan and Srinivasan (1998) and Srinivasan and Sathisan (1998). However, no attempt to integrate accessibility, market potential, and the location of TSFs has been documented in the literature.

\section{Objective}

The methodology presented here is intended to help select locations of TSFs based on an evaluation of transit market potential along a route. It aims to automate the procedure using GIS software. The methodology is based on establishing thresholds for the spatial extent of the market potential along a route, and quantifying the market potential at various locations of TSFs along the route. The spatial extent of the potential market is quantified based on walking distance and walking time considerations.

The analytical capabilities of ARC/INFO, a GIS program, have been employed to assess and analyze spatial data such as those for Traffic Analysis Zone (TAZ) including population by income and age groups, physically hand- 
icapped groups, etc. The data used for the case study were those available from the 1990 census.' An algorithm is proposed for this purpose. Steps 1 and 2 of this algorithm require a network analysis that is available in several popular GIS software packages (e.g., ARC/INFO, TransCad) used in transportation planning. Step 3 needs an overlay of data layers, a primary capability of any GIS software package. Once an index is defined in Step 4, a GIS package can be further used in determining the best locations and routes. For illustration purposes, a section of Route 101 of the CAT system is considered. This section traverses predominantly residential neighborhoods. The methodology presented aims at maximizing the potential transit ridership (i.e., the transit market potential).

In this article, walking time is used to measure accessibility. The first step would be to estimate the total population that has access to a TSF. Not all the population in the accessible region are likely to use the transit system. So, criteria have to be developed to estimate transit market potential for locating a TSF, specifically those that are not transfer/time-check points on a route.

\section{Problem Definition}

Consider a hypothetical TSF along a route in the transit network. The possibility that a resident in that locality will use the facility depends on several factors. These factors include age and gender of the person, income level, automobile ownership, employment, trip purpose, travel distance, physical mobility constraints, walking time (or distance) from the residence to the TSF, temporal considerations (e.g., time of the day, day of the week), access to other transport modes, and relative costs for using each mode.

The walking time to the TSF for the user is a measure of accessibility to the TSF. A potential user will not utilize the transit system if the walking time is very high (i.e., there is an upper limit to this walking time, whereas the lower limit can be as low as feasible, maybe a few seconds). For illustration purposes, consider five minutes to be the upper limit for the walking time (this may be changed depending on the actual situation). The walking time for two individuals traversing the same distance may be different (i.e., walking time is stochastic in nature). For simplicity, it is assumed that the walking speed is the 
same for all individuals, and it is about 1.2 meters per second ( 4 feet per second). The maximum walking distance for a user is the product of the threshold for walking time and the walking speed. Thus, for the case at hand, the maximum walking distance for a user will be equal to approximately 366 meters (1,200 feet). This is roughly 0.25 mile, which is a generally accepted premise of most transit operators. The accessible zone for this TSF is the area within the specified upper limit or threshold of walking time (or distance) from the TSF.

The creation of an accessible zone around the TSF is discussed later in this article. The zone is now defined to be accessible to all the residents in it. But, not all residents are likely to use the transit system. So, certain criteria have to be defined in order to identify the transit market potential. Using the estimated transit market potential for various TSF locations along a route, the objective is to find an optimal set of TSFs that maximize the transit market potential.

\section{Index of Transit Potential}

Transit-demand ridership depends on fare and other dummy variables (e.g., strikes) in addition to the factors identified in the previous section (McLeod et al. 1991). Neglecting fare and the dummy factors, the following demographic variables are significant in evaluating transit market potential:

- age group (less than 18 years and greater than 55 years)

- household income (less than $\$ 15,000$ per year)

- household size (greater than 3)

- household auto ownership (less than or equal to 1 )

- all unemployed persons

- all physically handicapped persons

These variables are presented solely for illustrative purposes. They may be changed or redefined, and other variables may be added as appropriate.

An index is constructed based on the potential captive riders for each of the variables presented above. This index, the Index of Transit Potential, is a measure of the transit market potential for each TSF. The ratio of the number of users based on a variable (one of the six defined above) who are within the 
accessible zone to the total value of the same variable in the study region multiplied by 1,000 (for normalization purposes) is first determined. The Index of Transit Potential is obtained by summing the ratios for each of the six variables described above. This can be mathematically expressed as shown in Equation 1 .

Index of Transit Potential $j=\Sigma_{i} \quad\left(\frac{n_{i j}}{N_{i}} \times 1000\right)$

where:

$j$ is the TSF number.

$i$ denotes categories such as unemployed, age, household size, vehicle ownership, physically handicapped, etc.

$n_{i j}$ is the number of users belonging to category $i$ in the region served by a TSF $j$.

$N_{i}$ is the total number of users belonging to category $i$ in the study region.

The value for each variable is defined to a scale of 1,000 . This value of 1,000 does not affect the TSF ranking (used in the selection process), and hence the selection process. All the variables are weighted equally, but, if warranted, individual weights can be assigned to each variable.

\section{Methodology}

Estimating the Index of Transit Potential means identifying the number of potential captive riders in the accessible zone. This requires spatial analysis that can be carried out using a GIS program. The following five steps are used in the process.

\section{Step 1: Simulate TSF Locations along a Route}

The street network of the study area is used as a starting point. This is represented as a "line" coverage in the GIS environment. Transit routes are also represented on this network. Along a desired route, TSF locations are simulated with a fixed distance between two adjacent TSFs. The TSFs are coded as a "point" coverage in the GIS environment. Once the TSF locations are simulated, the next step is to find the boundaries for the accessible zones. 


\section{Step 2: Define the Accessible Zone around a TSF}

In the second step, the capabilities of the GIS program are used to create a buffer around each simulated TSF. As an example, consider the previous discussion which noted that a user would at most walk for about 366 meters (1,200 feet). Using the "allocate" feature of the GIS program, a subnetwork (or allocated network) is created along the links near the TSF, joining all the points on the links that are at a distance of no more than 366 meters around each TSF. Thus, the walking distance from any point in this subnetwork to the TSF is either less than or equal to 366 meters. There are three possible ways of creating the accessible zone for measuring the accessibility to TSFs.

Method 1: Arc Lengths of Accessible Arcs Method. In the Arc Length method, it is assumed that the demographic criterion (or one of the six variables of interest to quantify transit market potential) is distributed proportionally to the lengths of the streets that are present in each TAZ. This assumes that the population distribution is proportional to the distribution of the street network within the TAZ - which is reasonable. The steps involved in delineating the demographic characteristics are:

1. Overlay the demographic coverage (TAZ) on the allocated coverage.

2. Estimate the lengths of "accessible" arcs for each TSF location that are present in each unit of the demographic coverage (TAZ).

3. Find the ratio of the accessible arc lengths to the total lengths of all arcs for each TAZ. This ratio is defined as the "arc ratio" for the TAZ. Thus, a percentage of the base unit of the demographic coverage is allocated for each TSF. This can be used for subsequent isolation of demographic data.

An illustration of a TSF location, the street network and TAZs near it, and portions of the links (arcs) on the street network that meet the criterion for accessibility (i.e., walking distance from the TSF is less than 366 meters) is shown in Figure 1(a). The Arc Length method has inherent advantages as it is relatively easy and eminently suited for implementation in a GIS environment. The disadvantage is that a particular segment of a route falling on a TAZ boundary is allocated to one of the two TAZs and not to both. 


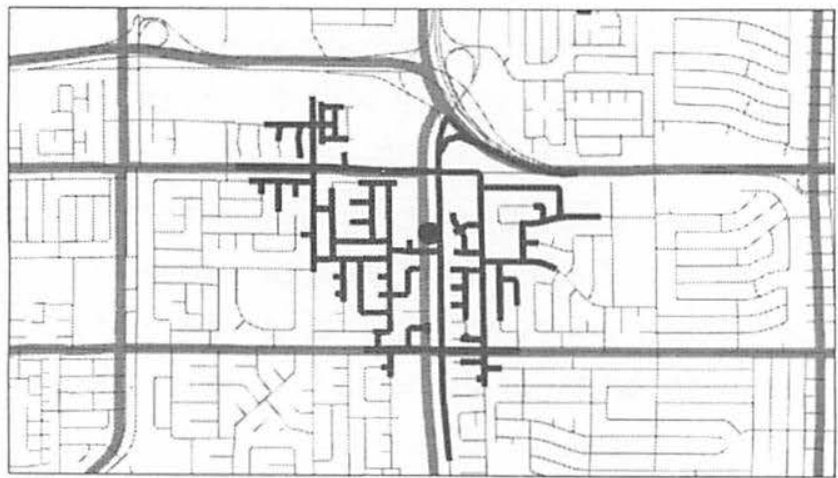

Legend

(a)

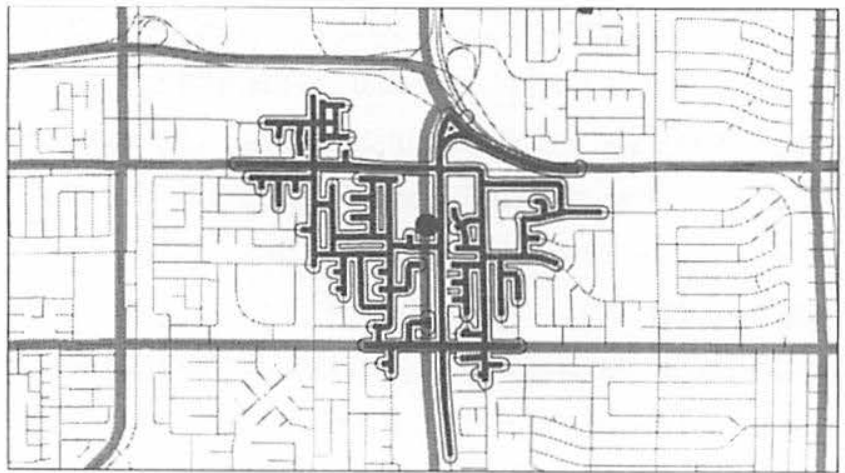

Buffer around accessible arcs

(b)

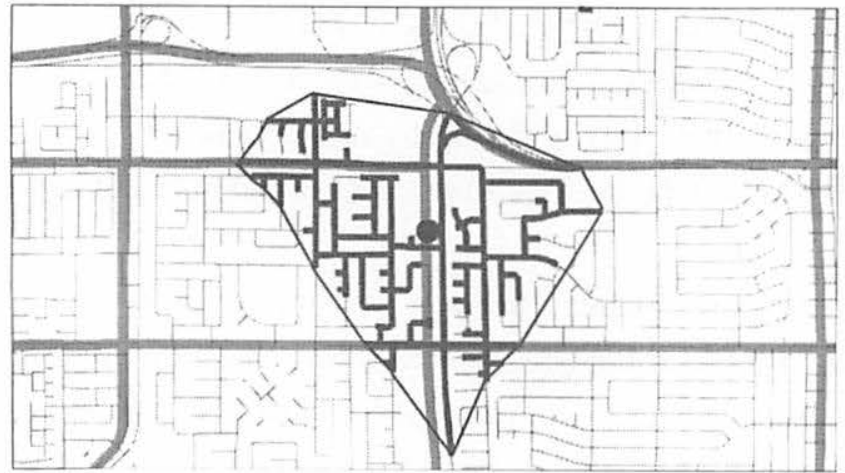

Hull polygon encompassing end points of accessible arcs

(c)

Figure 1. Regions around TSFs for measuring transit market potential 
Method 2: Buffer Around Accessible Arcs Method. The second method is to create a small buffer along the allocated arcs as shown in Figure 1(b). This assumes that the demographic variables are uniformly distributed throughout the TAZ. The steps involved in estimating data for each of the six variables of interest are:

1. Overlay the TAZ coverage on the allocated buffers.

2. Estimate the proportion of TAZs by area allocated to each TSF. This ratio is defined as the "buffer ratio."

3. Use this proportion to subsequently quantify data for each of the six demographic variables of interest.

This method solves the disadvantage of the first method, and thus helps correct the allocation of arcs to the TAZs. However, this approach raises other questions. For example, what if there was a huge apartment complex along the link (it cannot be divided into parts, as normally it is presumed that the TSF is accessible to the whole complex), or what if one of the arcs is identified as a freeway or the freeway passes through the polygon? These problems outweigh the benefits of solving the disadvantage of the Arc Length of Accessible Arcs method.

Method 3: Hull Polygon Method. The third method is a hull-type buffer shown in Figure 1(c). This is obtained by joining the farthest points of the accessible arcs as a polygon. The following steps are involved:

1. Overlay the TAZ coverage and identify the TAZs to which this polygon belongs.

2. Assuming that the demographic characteristics are uniformly distributed over these TAZs, the ratio of the area within the hull polygon to the total TAZ area is estimated and defined to be the "hull area ratio."

3. Estimates for each of the six demographic variables for each TAZ are evaluated as the product of the hull area ratio and the value of that variable for the TAZ. If the hull polygon encompasses more than one TAZ, the respective contribution of each TAZ is added to obtain the value for the TSF.)

But there are certain limitations as in the previous case, such as what if one of the arcs identified is a freeway, or if a freeway passes through the polygon. 
Any of the above approaches essentially involves a spatial overlay, which is easy to visualize in a GIS-based environment. The three types of spatial overlays can be performed using one of these data coverages:

- Overlay on land parcel coverage (obtained from the assessor's office).

- Overlay on census block data developed by the Census Bureau.

- Overlay on the TAZ coverage developed by the local metropolitan planning organization (i.e., the RTC in the Las Vegas metropolitan area).

The estimated measures reflect the level of accessibility of the simulated TSF to a population set. So, the final results obtained will reflect the level of detail or approximation used. The second and third types of overlay provide significant details required for the methodology. But, the street network coverage does not exactly mesh with the second and third types of coverage as the RTC developed these coverages from a different base source. Hence, due to the nature of available data, only the Arc Length of Accessible Arcs method is evaluated.

\section{Step 3: Estimate the Index of Transit Potential for Each TSF}

Consider the variables identified in the Index of Transit Potential section. Using the Census Transportation Planning Package (CTPP) report (U.S. DOT, FHWA 1995) developed for the TAZs, the number of users corresponding to each of the selected variables is estimated for each TAZ. From the proportion obtained in Step 2, the number of potential captive riders of each variable is allocated to each TSF. An illustration of this step is shown in Table 1. The first column in the table represents the TSF number, the second column is for TAZs for which this TSF is the closest, and the arc ratio for each TAZ is shown in the third column. Values for each of the six variables for the TAZ are shown in the next six columns; the last six columns show the contributions from each TAZ for each of the six variables (this is the product of the value of the variable for the TAZ and the arc ratio for the TAZ). The Index of Transit Potential for each TSF is calculated using Equation 1.

\section{Step 4: Locate Candidate TSFs}

The objective of this step is to locate the TSF locations along various routes. It is easy to simulate a large number of TSFs and select the optimal set 


\section{Table 1}

\section{Estimating Transit Market Potential Using Arc Lengths for Accessible Arcs Method}

\begin{tabular}{|c|c|c|c|c|c|c|c|c|c|c|c|c|c|c|}
\hline \multirow[t]{2}{*}{ TSF } & \multirow{2}{*}{$\begin{array}{c}\text { Contributing } \\
\text { TAZ }\end{array}$} & \multirow{2}{*}{ Ratio } & \multicolumn{6}{|c|}{ Variable Value for $T A Z^{a}$} & \multicolumn{6}{|c|}{ Variable Value for TSF ${ }^{a}$} \\
\hline & & & 1 & 2 & 3 & 4 & 5 & 6 & 1 & 2 & 3 & 4 & 5 & 6 \\
\hline \multirow[t]{4}{*}{83} & 409 & 0.18 & 22 & 4 & 28 & 1 & 13 & 14 & 3.92 & 0.71 & 5.02 & 0.22 & 2.35 & 2.44 \\
\hline & 410 & 0.07 & 11 & 5 & 58 & 25 & 27 & 53 & 0.77 & 0.34 & 4.08 & 1.77 & 1.88 & 3.74 \\
\hline & 423 & 0.29 & 17 & 8 & 10 & 19 & 22 & 5 & 4.94 & 2.19 & 2.89 & 5.55 & 6.43 & 1.45 \\
\hline & 424 & 0.12 & 59 & 37 & 16 & 31 & 25 & 26 & 7.06 & 4.46 & 1.95 & 3.67 & 2.97 & 3.12 \\
\hline \multicolumn{3}{|c|}{ Sum } & 109 & 54 & 112 & 76 & 87 & 98 & 16.69 & 7.70 & 13.94 & 11.21 & 13.63 & 10.75 \\
\hline \multirow[t]{3}{*}{90} & 443 & 0.08 & 28 & 22 & 49 & 32 & $\overline{55}$ & 63 & 0.08 & 2.21 & 1.76 & 3.95 & 2.57 & 4.38 \\
\hline & 444 & 0.22 & 6 & 5 & 7 & 8 & 6 & 2 & 0.22 & 1.42 & 1.02 & 1.46 & $1 . \overline{66}$ & 1.22 \\
\hline & \multicolumn{2}{|l|}{ Sum } & 34 & 27 & 56 & 40 & 60 & 65 & 3.63 & 2.78 & 5.41 & 4.23 & 5.60 & 5.46 \\
\hline
\end{tabular}

a. 1,2,3,4,5, and 6 represent age, income, unemployment, household size, physical mobility, and automobile ownership, respectively.

of TSFs for each route, based on the ranking given by the index value. A section of Route 101 of the CAT system is used to illustrate the procedure. Route 101 is about 20.7 kilometers $(68,000$ feet) long. The section selected is about 11 kilometers ( 36,000 feet) long and has 23 existing TSFs. But, instead of simulating 23 TSFs, 90 locations are simulated with a spacing of about 122 meters (400 feet). The 122-meter (400-foot) spacing is fixed, assuming that there will not be a significant difference in the ridership number if TSFs are located anywhere in between. In practice, based on the route conditions, spacing between TSFs can be made as small as necessary. Using the procedure discussed earlier, accessible zones are created for each of the simulated TSFs. For each simulated TSF, the potential captive riders based on the six variables and the Index of Transit Potential for each TSF are estimated. All the simulated TSFs are sorted based on the index values. Of these, locations with the highest index values are selected (subject to a minimum spacing between adjacent TSF locations) for providing the maximum accessible transit system along the route.

\section{Step 5: Select TSF Locations}

Since the methodology identifies the location of all the simulated TSFs, the spacing can be automatically determined. But, this depends on the number of TSFs selected along a route. A set of criteria is proposed for selecting the number of TSF locations and the spacing: 
1. Select all TSF locations with an index value greater than the mean index value for the route.

2. TSFs with ranks better or equal to the minimum number of TSFs required are selected.

3. The spacing between TSFs affects walking time as well as onboard riding time along a route, thus influencing transit demand.

None of these criteria consider spacing between TSFs. It can be logically interpreted that locating TSFs with very small spacing provides maximum accessibility. But, this is not economically nor operationally feasible. This stresses the need for solving the problem considering both accessibility and spacing between TSFs. This can be done by adding a constraint restricting spacing between two consecutive facilities.

\section{Case Study}

A section of Route 101 of the Las Vegas metropolitan area local transit system is considered for the case study. This section is 36,000 feet long. As discussed in Step 4, 90 TSF locations are simulated along this route with a fixed distance of about 122 meters ( 400 feet) between two consecutive TSFs. Points at a distance of about 366 meters are identified around each TSF. A typical street network that provides accessibility to a TSF is shown in Figure 1(a). This subnetwork, along with the street network, is overlayed on the TAZ coverage. The ratio of lengths of accessible arcs in each TAZ to the total lengths of all arcs lying in the TAZ is estimated for each TSF. The number of potential captive riders based on the selected demographic variables and Index of Transit Potential are estimated for each TSF.

The average index value obtained for the section (considering all 90 simulated stops) is 0.59 . If all the locations with index values greater than 0.59 were selected, then 17 TSF locations need to be provided. Since the maximum walking distance is set at 366 meters, the maximum spacing between two TSFs under ideal conditions is 731.5 meters (2,400 feet). A user located exactly between two TSFs has to walk a maximum distance.

Twenty-three TSF locations with the highest index values were selected along the route to provide exactly the same number of TSFs as exist currently. 
The accessibility region for the 23 selected TSF locations is shown in Figure 2 . Some stretches of the route have large spacing between stops. This simply reflects the spatial distribution of the variables used in the objective function.

The TSF identity (stop number), index values for each variable (age, income, unemployment, household size, mobility, and vehicle), and Index of Transit Potential for the existing and selected 23 locations are shown in Table 2. The locations are arranged in descending order based on the transit poten-

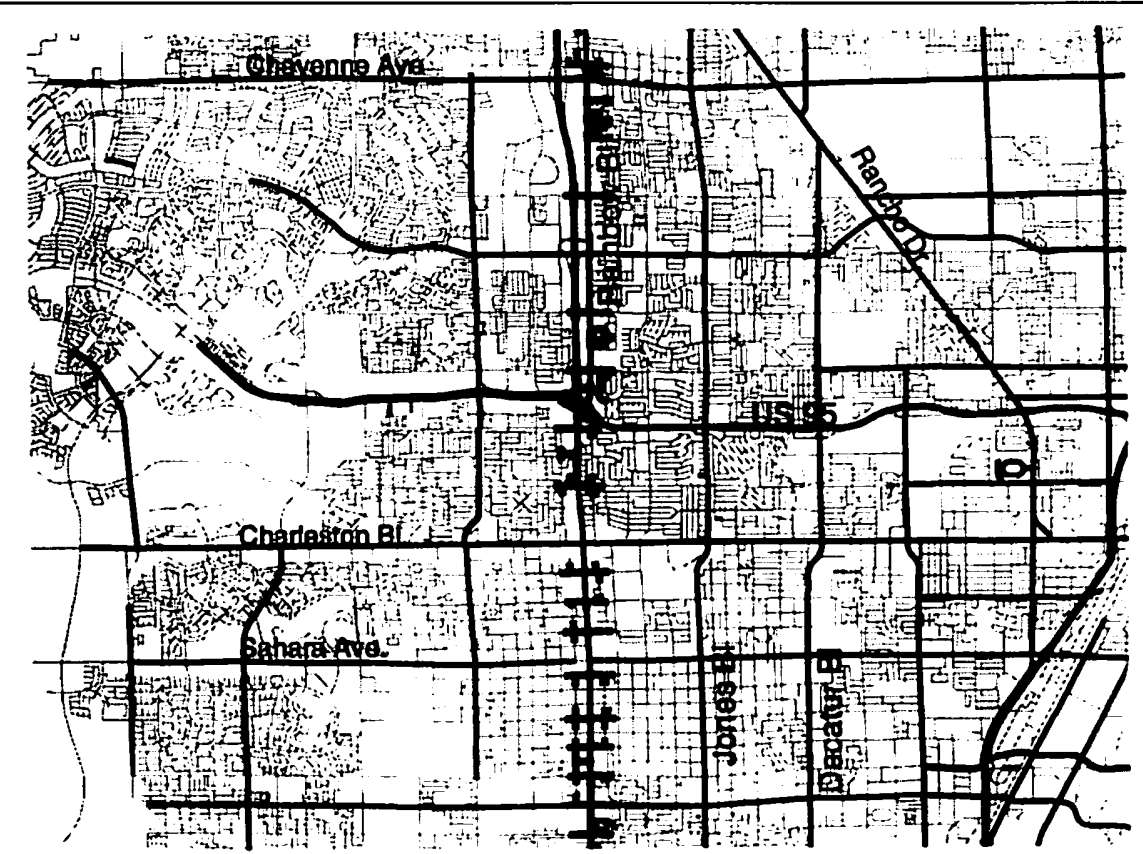

\section{Legend}

$\checkmark$ Major streets

Street network

Access to selected TSFs

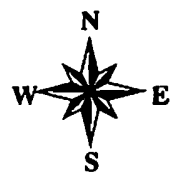

Figure 2. Accessible network for the selected TSF locations 
tial. The average index value for each variable and the Index of Transit Potential of the selected locations is greater than that of the existing locations. The highest Index of Transit Potential for an existing stop is observed as 11.48, whereas the highest Index of Transit Potential for a selected (or simulated) stop is 8.50. This is because the simulated stop does not include the existing stop with the highest Index of Transit Potential. This can be taken care of by simulating bus stops with small spacing.

\section{Discussion}

The results obtained from this study are different from those obtained from the procedure used by the local transit authorities. This may be due to the following:

- The case study uses only data related to residential locations for selecting the TSFs. But, other vital land-use aspects such as commercial centers and offices were not accounted for in the analysis. However, they can easily be incorporated as TAZ attributes.

- Socioeconomic and political aspects were not addressed.

- The approach does not consider data at microscopic levels. Thus, finally it might result in locating the TSF exactly in the middle of an intersection. This may be an optimal location from a market potential point of view, but it may not be feasible for practical reasons. The normal procedure in the Las Vegas area is to opt for farside locations at the intersection. But, this may result in a different index value for the TSF.

- Another aspect ignored in the study is route spacing. "The route spacing and route length though have unique values over time, will have a significant affect on the passenger access time," state Chang and Schonfeld (1995). This problem can be taken care of by simultaneously considering all the routes, with all TSF locations in the transit system.

The case study demonstrates that the methodology presented is a useful decision-support tool for transit system operators. Transit demand depends on the spatial distribution of potential users along a route. Hence, the spacing between TSFs should vary along a route. The methodology presented selects TSF locations based on the Index of Transit Potential. However, various other 
Table 2

Index Values for Each Category and Accessibility Index for TSF Locations

\begin{tabular}{|c|c|c|c|c|c|c|c|}
\hline $\begin{array}{l}\text { Stop } \\
\text { No. }\end{array}$ & $\begin{array}{c}\text { Age } \\
\text { Index }\end{array}$ & $\begin{array}{l}\text { Income } \\
\text { Index }\end{array}$ & $\begin{array}{c}\text { Unemployment } \\
\text { Index }\end{array}$ & $\begin{array}{c}\mathrm{HH} \\
\text { Index }\end{array}$ & $\begin{array}{l}\text { Mobility } \\
\text { Index }\end{array}$ & $\begin{array}{l}\text { Vehicle } \\
\text { Index }\end{array}$ & $\begin{array}{l}\text { Access } \\
\text { Index }\end{array}$ \\
\hline \multicolumn{8}{|c|}{ Existing Stops } \\
\hline 23 & 1.46 & 2.11 & 2.79 & 1.73 & 2.08 & 1.32 & 11.48 \\
\hline 10 & 0.62 & 1.05 & 0.88 & 0.75 & 0.90 & 0.61 & 4.82 \\
\hline 11 & 0.88 & 0.63 & 0.55 & 0.72 & 0.57 & 1.04 & 4.38 \\
\hline 12 & 0.69 & 0.40 & 0.42 & 0.67 & 0.43 & 0.80 & 3.42 \\
\hline 13 & 0.38 & 0.21 & 0.19 & 0.25 & 0.21 & 0.44 & 1.68 \\
\hline 12 & 0.42 & 0.16 & 0.15 & 0.24 & 0.19 & 0.49 & 1.64 \\
\hline 9 & 0.25 & 0.14 & 0.21 & 0.36 & 0.17 & 0.32 & 1.46 \\
\hline 1 & 0.18 & 0.22 & 0.17 & 0.14 & 0.13 & 0.21 & 1.05 \\
\hline 17 & 0.31 & 0.14 & 0.13 & 0.08 & 0.19 & 0.12 & 0.97 \\
\hline 3 & 0.21 & 0.08 & 0.09 & 0.17 & 0.08 & 0.29 & 0.91 \\
\hline 2 & 0.19 & 0.09 & 0.09 & 0.15 & 0.08 & 0.26 & 0.84 \\
\hline 16 & 0.26 & 0.12 & 0.11 & 0.06 & 0.16 & 0.10 & 0.81 \\
\hline 20 & 0.13 & 0.14 & 0.10 & 0.18 & 0.10 & 0.13 & 0.77 \\
\hline 21 & 0.13 & 0.14 & 0.10 & 0.17 & 0.10 & 0.13 & 0.77 \\
\hline 4 & 0.15 & 0.06 & 0.06 & 0.12 & 0.06 & 0.22 & 0.67 \\
\hline 18 & 0.19 & 0.09 & 0.09 & 0.06 & 0.12 & 0.07 & 0.63 \\
\hline 19 & 0.14 & 0.10 & 0.09 & 0.10 & 0.10 & 0.09 & 0.61 \\
\hline 5 & 0.10 & 0.08 & 0.05 & 0.08 & 0.06 & 0.12 & 0.49 \\
\hline 15 & 0.10 & 0.07 & 0.06 & 0.05 & 0.07 & 0.04 & 0.39 \\
\hline 14 & 0.02 & 0.02 & 0.02 & 0.02 & 0.02 & 0.01 & 0.12 \\
\hline 7 & 0.01 & 0.02 & 0.01 & 0.01 & 0.01 & 0.01 & 0.08 \\
\hline 6 & 0.00 & 0.00 & 0.00 & 0.00 & 0.00 & 0.01 & 0.02 \\
\hline 8 & 0.00 & 0.00 & 0.00 & 0.00 & 0.00 & 0.00 & 0.00 \\
\hline Average & 0.30 & 0.26 & 0.28 & 0.27 & 0.25 & 0,30 & 1.65 \\
\hline \multicolumn{8}{|c|}{ Selected Stops } \\
\hline 89 & 0.90 & 2.01 & 1.04 & 0.83 & 1.51 & 2.21 & 8.50 \\
\hline 87 & 0.69 & 0.58 & 0.83 & 0.63 & 0.83 & 1.01 & 4.57 \\
\hline 90 & 0.53 & 0.95 & 0.53 & 0.40 & 0.93 & 1.10 & 4.45 \\
\hline 40 & 0.49 & 1.00 & 0.52 & 0.44 & 0.82 & 0.76 & 4.03 \\
\hline 41 & 0.65 & 0.49 & 0.64 & 0.78 & 0.42 & 0.43 & 3.42 \\
\hline 36 & 0.42 & 0.63 & 0.51 & 0.43 & 0.57 & 0.56 & 3.12 \\
\hline 84 & 0.45 & 0.25 & 0.43 & 0.53 & 0.28 & 0.28 & 2.22 \\
\hline 38 & 0.19 & 0.63 & 0.20 & 0.13 & 0.46 & 0.42 & 2.03 \\
\hline 43 & 0.40 & 0.25 & 0.28 & 0.47 & 0.24 & 0.23 & 1.87 \\
\hline 50 & 0.36 & 0.22 & 0.24 & 0.42 & 0.22 & 0.20 & 1.64 \\
\hline 33 & 0.27 & 0.15 & 0.42 & 0.36 & 0.16 & 0.23 & 1.60 \\
\hline 88 & 0.12 & 0.21 & 0.17 & 0.09 & 0.22 & 0.31 & 1.12 \\
\hline 7 & 0.24 & 0.09 & 0.19 & 0.34 & 0.09 & 0.10 & 1.06 \\
\hline 32 & 0.14 & 0.08 & 0.23 & 0.19 & 0.08 & 0.12 & 0.85 \\
\hline 46 & 0.20 & 0.05 & 0.10 & 0.23 & 0.07 & 0.05 & 0.70 \\
\hline 29 & 0.13 & 0.07 & 0.15 & 0.14 & 0.10 & 0.11 & 0.70 \\
\hline 60 & 0.20 & 0.09 & 0.05 & 0.08 & 0.12 & 0.08 & 0.61 \\
\hline 42 & 0.10 & 0.07 & 0.10 & 0.12 & 0.06 & 0.06 & 0.51 \\
\hline 64 & 0.15 & 0.07 & 0.04 & 0.06 & 0.10 & 0.06 & 0.48 \\
\hline 1 & 0.05 & 0.16 & 0.04 & 0.03 & 0.08 & 0.10 & 0.46 \\
\hline 16 & 0.08 & 0.08 & 0.07 & 0.10 & 0.06 & 0.05 & 0.45 \\
\hline 5 & 0.10 & 0.04 & 0.08 & 0.14 & 0.04 & 0.04 & 0.43 \\
\hline 77 & 0.07 & 0.07 & 0.10 & 0.07 & 0.05 & 0.06 & 0.42 \\
\hline Average & 0,30 & 0,36 & 0,30 & 0.31 & 0,33 & 0.37 & 1.97 \\
\hline
\end{tabular}


aspects related to operations should also be considered in the final solution. This research opens up avenues to examine ways of estimating the transitdemand origin matrix based on land-use patterns; demographic characteristics of the region; and demand variation over time of day, month, and year. Further, the functional classification of roads should be incorporated in the analysis so as to eliminate freeways and other facilities (that do not permit pedestrian or bicycle traffic) from the accessible network.

\section{Conclusions}

The objective of this study was to define a measure for accessibility to each TSF and use the measure to identify optimal locations for TSFs. An Index of Transit Potential, a measure for accessibility, is defined based on the potential captive riders belonging to various demographic variables. A methodology to identify locations for TSFs along various routes was proposed and its use was demonstrated. It is best suited to locate general TSFs (i.e., those that are not time-check points or transfer points). The need for spatial analysis shows the increasing emphasis in solving problems easily using GIS-based environments. The methodology presented serves as a good decision-support tool for designing and operating transit systems. Thus, the final decisions on TSF locations should also consider other factors from an operations point of view.

\section{Acknowledgments}

The authors thank the staff at the RTC of Clark County, Nevada, for providing data and information that were essential for this work. Further, they thank two anonymous referees for their valuable comments.

They also acknowledge a grant from TRW Environmental Safety Systems, Inc., which provided partial support for this work.

\section{Endnote}

1. The Las Vegas metropolitan area has seen a tremendous amount of change in population in the last decade; however, this does not have any bearing on the proposed methodology. 


\section{References}

Bach, L. 1981. The problem of aggregation and distance for analysis of accessibility and access opportunity in location allocation models. Environment and Planning $A$ 13: 955-978.

Chang, S. K., and P. M. Schonfeld. 1995. Optimal dimensions of bus service zones. ASCE Journal of Transportation Engineering 119(4): 567-585.

Chu, Xuehao. 1998. 1995 NPTS analyzed to develop information base on public transit. CUTRlines 9(3): 1-6.

Environmental Systems Research Institute, Inc. 1997. Understanding GIS-The ARC/INFO method. New York: John Wiley Sons, Inc.

Gomez, A., and F. Zhao. April 1998. Improving transit access estimate in transportation modeling using GIS. Paper presented at the 11 th annual GIS for Transportation Symposium. Salt Lake City, Utah.

Henk, R. H., and S. M. Hubbard. 1996. Developing an index of transit service availability. Transportation Research Record 1521: 12-19.

Johnston, R. J. 1966. An index accessibility and its use in the study of bus services and settlement patterns. Journal of Economic and Social Geography 57: 33-38.

McLeod Jr., M. S., K. J. Flannelly, L. Flannelly, and R. W. Behnke. 1991. A multivariate, time series model of transit ridership based on historical aggregate data: The past, present, and future of Honolulu. Paper submitted for 70th annual meeting of the Transportation Research Board. Washington, DC.

O’Neill, W. A., D. R. Ramsey, and J. Chou. 1992. Analysis of transit service areas using geographic information systems. Transportation Research Record 1364: 131-138.

Regional Transportation Commission of Clark County. 1997. Citizens Area Transit: Bus stop guidelines. Las Vegas, NV: Planning Division.

Sathisan, S. K., and Nanda Srinivasan. 1998. Evaluation of accessibility of urban transportation networks. Transportation Research Record 1617: 78-83.

Srinivasan, N., and S. K. Sathisan. May 1998. Quantification and analysis of transportation system accessibility. Proceedings of the Transportation, Land-Use and Air Quality: Making the Connection Conference, Sponsored by the American Society of Civil Engineers. Portland, Oregon, 208-217.

U.S. Department of Transportation, Federal Highway Administration. 1995. CTPP handbook: An instructional guide to the 1990 Census Transportation Planning Package, FHWA-PD-95-019. 


\section{About the Authors}

SRINIVAS S. Pulugurtha (pss@trc.unlv.edu) is an assistant research professor at the Transportation Research Center, University of Nevada, Las Vegas. He received his Ph.D. in civil engineering (focus in transportation) from the University of Nevada, Las Vegas in 1998.

Shashi S. Nambisan (shashi@ce.unlv.edu) is a professor of civil engineering and director of the Transportation Research Center at the University of Nevada, Las Vegas.

NANDA SRINIVASAN (n_18Ø@yahoo.com) is a GIS programmer for Cambridge Systematics. He earned his M.S.E. from the University of Nevada, Las Vegas in 1997. His current efforts include work on the Census Transportation Planning Package for 2000. 